1

2

3

4

5

6

7

8

9

10

11

12

13

14

15

16

17

18

19

20

21

22

23

24

\title{
Dissecting the microenvironment around biosynthetic scaffolds in murine skin wound healing
}

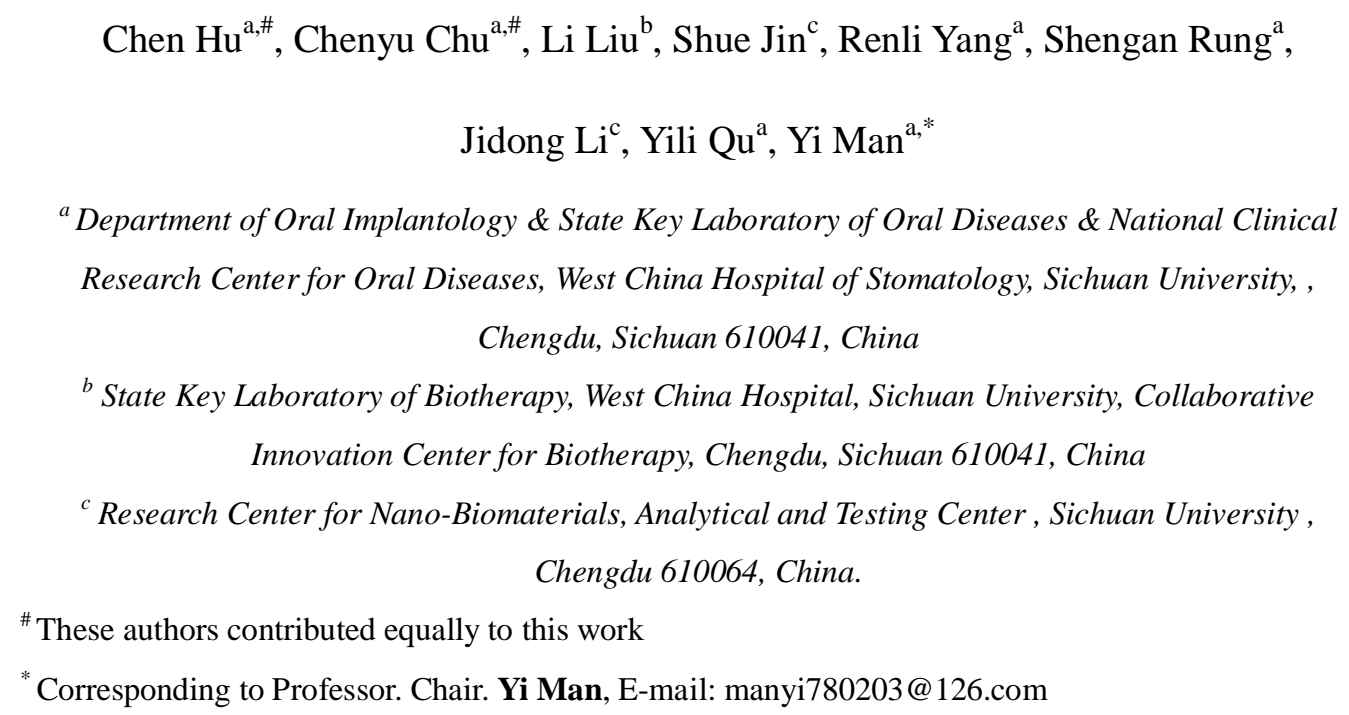


Abstract: Structural properties of biomaterials play critical roles in guiding cell behaviors and influence the immune response against them. We fabricated electrospun membranes with three types of surface topography (Random, Aligned, and Latticed). The aligned membranes showed immunomodulatory ability, and led to faster wound healing, reduced fibrotic response and enhanced regeneration of cutaneous appendages when used in skin wound repair. Based on that, we performed single-cell RNA sequencing analysis on cells from wounded mouse skin in the presence or absence of the Aligned scaffold. Keratinocytes, fibroblasts, and immune cells including neutrophils, monocytes, macrophages, dendritic cells, and $\mathrm{T}$ cells showed diverse cellular heterogeneity. More hair follicle progenitor cells, inner root sheath cells (anagen-related) and fibroblast subsets were found in the Aligned group, which corresponded to the improved regeneration of hair follicles and faster wound closure in the presence of scaffold. Immune responses towards the biomaterial differed from that of control group. In aligned samples, infiltrated macrophages and neutrophils were reduced, whereas more effector $\mathrm{T}$ cells were recruited. The time course of immune response was possibly advanced towards an adaptive immunity-dominant stage by the scaffold. The microenvironment around scaffold involved intricate interplay of immune cells and cutaneous cells, and wound healing was the comprehensive results of numerous influencing factors working together.

Key words: Surface topography, immune microenvironment, immunomodulatory ability, cellular heterogeneity 


\section{Introduction}

48 Biomaterials and devices implanted in the body have a broad spectrum of clinical

applications including tissue regeneration, cell transplantation, controlled drug release, and continuous monitoring of physiological conditions $(1,2)$. Among the components of a cell microenvironment, structural features (macroscale, microscale and nanoscale features) play critical roles in guiding cell behaviors (3). Electrospinning technology has been widely applied in preparing scaffolds due to its simplicity, capacity to form fibers on the micro- and nanoscale, structural control of electrospun membranes and cost-effectiveness (4). Electrospun nanofibers could be assembled into well-ordered nanofiber meshes with different morphology, e.g. parallel alignment or latticed patterns of nanofibers $(5,6)$, and have been preferentially applied to the regeneration of diverse tissues like skin and bone $(6,7)$. Upon implantation of a material, cells of both the innate and adaptive immune system have a role in the host response (8). Previous studies illustrated the type 1 (pro-inflammatory) immune polarization driven by $\mathrm{T}$ helper 1 (Th1) cells, and the induced pro-inflammatory M1 activation of macrophages (stimulated by IL-2 and IFN- $\gamma$ from Th1 cells). By contrast, in type 2 immune response, T helper 2 (Th2) cells produce cytokines like IL-4 and IL-13, which regulate the polarization of macrophages towards an anti-inflammatory M2 activation $(9,10)$. More recently, a type 17 immune response was reported to promote chronic fibrosis in tissue around implants $(11,12)$. In summary, previous studies usually focused on certain types of immune cells, like macrophages, and explored their roles in the host response. However, the immune response is jointly regulated by 
various immune cells, whose phenotype and function are dictated by external and internal signals. An overview of different immune cells in the microenvironment will aid in comprehensive understanding of the immune responses elicited by scaffolds. Technological advances such as single-cell RNA sequencing (scRNA-seq) $(13,14)$ have enabled cell populations, functions, and the nuances of their phenotypes in vivo to be studied at a high resolution. By changing the collector, we developed poly(lactic-co-glycolic acid)-fish collagen (PLGA-FC) hybrid electrospun scaffolds with three types of surface topography, i.e. the group with randomly oriented fibers (Random group), the group with mesh-like topography in macroscale and randomly oriented fibers in microscale (Latticed group), and the group with aligned fibers (Aligned group). We explored the regenerative outcomes of these scaffolds in rat/mouse dorsal skin excisional wounds, and evaluated their immunomodulatory properties. The scaffold with the best performance was further investigated. Microenvironment around the scaffold was probed by scRNA-seq. Heterogeneity of keratinocytes, fibroblasts, and immune cell populations, cellular functions, and their interactions in vivo were explored.

\section{Results}

\section{Evaluation of wound healing in a rat skin wound model}

We fabricated scaffolds with random, aligned and latticed patterns of fibers (Fig. 1A), and placed them below the full-thickness excisional wound (diameter $=6 \mathrm{~mm}$ ) on rat dorsal skin (SD rat). The Ctrl group received no scaffolds (Fig. 1C). Biophysical properties of the scaffolds were summarized in Fig. S1. Workflow for evaluating 
91 wound healing was summarized in Fig. 1B. Wound healing rate was significantly accelerated by the aligned membranes (Fig. 1D-E). The Latticed group showed delayed wound healing with the largest residual wound area left at day 7. On day 14, basically all groups achieved complete closure of the wound. Above the scaffolds, surrounding epithelium formed epithelial tongue as the first layer advancing towards the wound (15). On day 7, the Aligned group presented the fastest coverage of the wound, leaving the smallest gap width, whereas the latticed membrane seemed to impede the advancement of surrounding epithelium (Fig. 1F-G, Fig. S2). On day 14, re-epithelialization was completed in all groups except for the Latticed group. All groups showed matured stratified epithelium (Fig. 1F). Immunofluorescent staining for Krt5 (keratin secreted by keratinocytes in the basal layer) and Krt10 (keratin secreted by differentiated keratinocytes in the supra-basal layers) showed that on day 7, the Aligned group had the largest area of newly formed keratinized epithelium, and more keratinocytes were undifferentiated (Krt5-positive). On day 14, all groups revealed formation of stratified epithelium (Fig. 1H-I) (15). For the dermis part, wound space below epithelium was filled with granulation tissue on day 7. Collagen kept being deposited and remodeled (Fig. S2). On day 28, the Aligned group had more regenerated hair follicles and sebaceous glands than other treatment groups (Fig. 1F). The scaffolds were placed subcutaneously in rats to evaluate host response against them. Thickness of fibrotic capsules around scaffolds was measured at day 3, 7 and 14. The Aligned group had the smallest fibrotic capsule thickness at all time points (Fig. 1J-K, Fig. S3). The bulk-tissue RNA-Seq analysis for samples harvested 
113

114

115

116

117

118

119

120

121

122

123

124

125

126

127

on day 7 ( $\mathrm{n}=3$ for each group) identified transcripts corresponding to 34459 genes, distributed over 6 orders of magnitude of expression level. Principal component analysis (PCA) of the data revealed that gene expression profiles of the Random and Aligned samples were more similar, compared to that of Latticed and Ctrl samples (Fig. 1L). In Gene ontology (GO) analysis, random and aligned scaffolds induced up-regulated expression of genes associated with immune responses when compared with the Ctrl samples, whereas the Latticed group did not (Fig. 1M). Kyoto Encyclopedia of Genes and Genomes (KEGG) analysis also revealed gene enrichment in an immune-related pathway "Cytokine-cytokine receptor interaction" (KEGGID rno04060) (Fig. S4A-B) (16). Real-time Quantitative Polymerase Chain Reaction (qPCR) confirmed these elevated genes observed in Aligned and Random groups (Fig. S4C). The data suggested that random and aligned membranes were able to modulate the local immune microenvironment. To explore whether the scaffolds had similar performance in other species, we applied them in mouse skin wound model.

\section{Wound healing in mouse}

Workflow for evaluating wound healing in C57BL/6 mouse was summarized in Fig. 2A. We placed three types of scaffolds below the full-thickness excisional wound (diameter=6mm) $($ Fig. 2B). The Ctrl group received no scaffolds. Wound coverage was faster in the Aligned (residual wound area=29.29 $\pm 4.81 \%$ ) and Ctrl group $(20.80 \pm 4.66 \%)$ on day 7 . On day 14 , basically all groups achieved complete closure of the wound (Fig. 2C-D). The Aligned group had the smallest gap width on day 7 , followed by the Ctrl group (Fig. 2E-F). On day 14, re-epithelialization was completed 
135 in all groups except for some samples of the Latticed group. All groups showed

136 matured stratified epithelium. Regeneration of hair follicles was seen in Aligned

137 samples after 14 days of healing (Fig. 2E). Scaffolds were also placed subcutaneously

138 to evaluate the host response against them. The aligned membranes had the smallest

139 capsule thickness over the observation period (Fig. 2G, Fig. S5). Bulk-tissue

140 RNA-Seq for Aligned and Ctrl samples harvested on day 7 ( $\mathrm{n}=3$ for each group) was

141 performed. Compared with Ctrl, the Aligned group showed increased genes enriched

142 in inflammatory response, leukocyte chemotaxis and migration (Fig. 2I). KEGG

143 analysis revealed elevated gene expression in immuno-related signaling pathways for

144 the Aligned group (KEGGID mmu04657, mmu04668, mmu04060 and mmu04064)

145 (Fig. 2J). To summarize, in both rat and mouse models, the aligned membranes led to

146 faster wound healing, reduced fibrotic response and enhanced regeneration of

147 cutaneous appendages compared to other membranes. Meanwhile, they showed

148 immunomodulatory properties. We therefore sought to explore how aligned

149 membranes regulated peri-implant microenvironment. Here, we used scRNA-seq to

150 sequence cells from the wounded murine full-thickness skin 7 days post-wounding.

151 Single-cell transcriptome analysis of full-thickness skin after wounding and

\section{2 scaffold placement}

153 We isolated cells from the Aligned and Ctrl samples $(n=4$ biological replicates in each

154 group), and applied them to the 10X scRNA-seq platform (Fig. 3A). A total of 8,982

155 cells in the Ctrl group and 9,593 cells in the Aligned group were captured. After cell

156 filtering, 17,181 single cell transcriptomes were included in the final dataset $(8,869$ 
157 for the Aligned group and 8,312 for the Ctrl group) (Fig. S6). We first

158 computationally pooled cells from Ctrl and Aligned groups to create a virtual

159 aggregate. Unsupervised clustering using Seurat categorized the cells into 26 clusters

160 based on global gene expression patterns (Fig. S7), which were assigned to thirteen

161 main classes of cells (Fig. 3B): keratinocytes (KER), fibroblasts (FIB), sebocytes

162 (SEB), smooth muscle cells (SMC), endothelial cells (EC), Schwann cells (SC),

163 melanocytes (MEL), innate lymphoid cells (ILC), monocyte-macrophages (MAC), T

164 cells (TC), neutrophils (NEU), dendritic cells (DC) and B cells (BC). Marker genes

165 for each cell cluster were shown in the heatmap (Fig. 3C). The composition of each

166 cell cluster was listed so that the proportion of cells from two groups could be

167 identified across all cell clusters (Fig. 3B). As smooth muscle cells (57\% from the

168 Aligned group and $43 \%$ from the Ctrl group) could hardly regenerate at 7 days

169 post-wounding, we regarded a proportion within $50 \pm 7 \%$ as equilibrium between two

170 groups. Genes related to macrophages (Itgam, Cd68, Arg1, Mrc1) were significantly

171 higher expressed by the Ctrl samples (Fig. 3D).

172 Sub-clustering of keratinocytes reveals a higher proportion of hair follicle 173 progenitor cells from the Aligned group

174 We selected cells that were in the first-level clustering defined as keratinocyte, and

175 subjected them to a second round of unsupervised clustering (Fig. 4A). Terminal

176 cornification of epidermis is achieved by keratinocytes passing through basal layers,

177 differentiated layers, and cornified layers (15). In our study, the inter-follicular

178 epithelium was composed of the basal layer cells (IFEB, Krt5 ${ }^{\text {hi }} \mathrm{Krt} 14^{\mathrm{hi}}$ ), supra-basal 
179 layer cells $\left(\mathrm{Krt10} 0^{\mathrm{hi}} \mathrm{Krt}^{\mathrm{hi}}\right.$ IFED1, Krt10 ${ }^{\mathrm{hi}} \mathrm{Mt} 4^{\mathrm{hi}}$ IFED2), and cornified layer (Lor ${ }^{+}$

180 IFED3) cells (Fig. 4A-B). The location of keratinocyte subsets was marked in Fig. 4C.

181 Hair follicles in this study were in second telogen since the mice were 8 to 10 weeks

182 old when they were sacrificed (17). However, anagen hair follicle gene signatures

183 were also found, possibly resulting from hair follicle regeneration after wounding.

184 Upper hair follicle cells were separated into three subsets named uHF1

$185\left(\mathrm{Krt79}{ }^{\mathrm{hi}} \mathrm{Krt} 17^{\mathrm{hi}}\right), \mathrm{uHF} 2\left(\mathrm{Klk} 10^{+} \mathrm{Krt} 79^{\mathrm{hi}}\right)$, and uHFB (the upper hair follicle basal layer

186 cells, Sostdc $1{ }^{\text {hi }}$ Apoe $\left.{ }^{\text {hi }}\right)$. The hair follicle progenitor cells (Krt28 ${ }^{\text {hi }}$ Lhx $2{ }^{\text {hi }}$ Mki67 ${ }^{\text {hi }}$ HFP)

187 were highly proliferative. Germinative layer cells $\left(\mathrm{Mt}^{\mathrm{hi}} \mathrm{Dcn}^{\text {hi }}\right)$ belonging to anagen

188 hair follicles also expressed high cell proliferation related genes like Top2a and Birc5.

189 Meanwhile, they had a basal cell gene signature $\left(\mathrm{Krt} 5{ }^{\mathrm{hi}} \mathrm{Krt} 14^{\mathrm{hi}}\right)$. The inner root sheath

190 (IRS) and cortex cells were characterized by Krt28, Krt27, Krt73, and Krt25, markers

191 for the Henle and Huxley layers of anagen hair follicles (18). Cells of the inner bulge

192 layer (IB) and outer bulge layer (OB) expressed their typical gene signatures (Fig.

193 4A-C). When analyzing the inter-group differences, the Aligned group contributed to

194 a larger proportion of hair follicle progenitor cells (HFP) and highly proliferative

195 inner root sheath cells (IRS) (Fig. 4A). The overall difference analysis also revealed

196 up-regulation of genes (Krt28, Sox18) related to hair follicle stem cells in the Aligned

197 group (Fig. 3D). This might explain the enhanced hair follicle regeneration observed

198 in Aligned groups either on rat or mouse models.

199 Inter-group differences in fibroblasts suggest more active ECM formation in the

200 Aligned group 
201 The dermis consists of several layers: the papillary dermis lies closest to the epidermis,

202 the underlying reticular dermis contains the bulk of the fibrillary extracellular matrix,

203 and beneath the reticular dermis lies the hypodermis (19). Fibroblasts from different

204 layers presented distinct gene signatures (Fig. 4D). There were four populations of

205 papillary fibroblasts $\left(\mathrm{Crabp}^{+} \mathrm{Col} 23 \mathrm{a} 1^{+}\right)(20,21)$. Dermal fibroblasts expressed

206 increased Ccl11 and Dcn. The Gpx $3^{+} \mathrm{Plac} 8^{\text {hi }}$ subset was identified as hypodermis

207 fibroblast located to the adventitia (HDF1), and the $\mathrm{Gpx} 3^{+} \mathrm{Plac} 8^{\text {lo }}$ subset (HDF2) was

208 around the panniculus carnosus muscle (18). Contractile myo-fibroblasts (Acta2 ${ }^{+} \mathrm{MF}$ )

209 expressed elevated genes associated with cell proliferation $\left(\right.$ Birc $\left.5^{\text {hi }}\right)$. Composition of

210 each subset revealed that more papillary fibroblasts (PF2), dermal fibroblasts (DF),

211 hypodermis fibroblasts (HDF2) and myo-fibroblasts (MF) were from the Aligned

212 group, suggesting more robust ECM formation in the presence of scaffolds.

213 Neutrophils and dendritic cells are more abundant in the Ctrl samples

214 After wounding, neutrophils close to the focus of injury migrate toward the nidus,

215 followed by those recruited from more than $200 \mu \mathrm{m}$ from the site of tissue injury (22).

216 Three neutrophil subsets were identified in our study (Fig. 4E). Peripheral blood

217 neutrophils (PBN) expressed typical gene signatures including Csf3r, Pglyrp1, Il1b,

218 and Retnlg. Neutrophil 2 (Neu2) expressed elevated $\mathrm{Cd} 14$ and $\mathrm{Ccl} 3$, and was

219 identified as antimicrobial phagocytic neutrophils. Neutrophil 1 (Neu1) expressed

220 elevated Ccr1, a chemokine receptor that mediates neutrophil migration.

221 Correspondingly, PBN showed gene enrichment in leukocyte chemotaxis and

222 migration; Neu1 expressed genes enriched in leukocyte/granulocyte chemotaxis and 
migration, and Neu2 was enriched in anti-bacterial biological processes (Fig. 4F).

According to the correlation analysis, Neu 1 and Neu 2 were derived from PBN in circulation (Fig. 4G). In Neu1 and Neu2, more cells belonged to the Ctrl group (Fig.

4E), suggesting more significant Neu1 and Neu2 infiltration in the Ctrl samples at a

proliferative stage (7 days post-wounding). Dendritic cells were classified into two subpopulations (Fig. 4H). The subset derived from monocytes (DC) was characterized by increased $\mathrm{Cd} 207$ and $\mathrm{Cd} 86(10,23)$. The Langerhans cell subset bore a keratinocyte gene signature, probably transferred from the resident microenvironment (Fig. 4I) (24). Both DC and LC presented elevated major histocompatibility complex (MHC) molecules, suggesting an antigen presenting function of them (Fig. 4J). LCs contained more Ctrl-derived cells, indicating differences in Langerhans cell infiltration between groups.

\section{Macrophage heterogeneity and their down-regulation by scaffolds}

To explore the heterogeneity of macrophages in vivo and their inter-group differences, we subjected them to further unsupervised sub-clustering. Four subsets were determined (Fig. 5A). The subset that showed increased anti-inflammatory genes (Ccl8, Folr2, C1qa and Mrc1) was named anti-inflammatory macrophages (AIM) (25,

(Ptgs2, Ccl3, Inhba, Nos2) was named pro-inflammatory macrophages (PIM) (27, 28).

242 The monocyte subset (Mono) showed higher Ly6c2, Plac8, Cd14, and Clec4e, 243 indicting inflammatory responses against lesions and microorganism $(29,30)$. The 
245 (M-DC) (Fig. 5B) (31). We further found that canonical M1 and M2 markers were not

246 entirely consistent with computationally determined AIM and PIM. Arg1, a canonical

247 M2 marker, was expressed by basically all monocyte-macrophage subsets (AIM, PIM,

248 Mono), and was regarded as a pan-macrophage marker in this study (Fig. 5B).

249 Expression of another type 2 gene, Socs3, did not parallel Mrc1 expression either.

250 Similar disparity was found in the expression of canonical type 1 genes. M-DC, rather

251 than PIM, expressed more Cd86. Neither was the expression of Cd86 correlated with

252 Nfkbiz. Expression of other genes associated with fibrotic or regenerative

253 macrophage subsets in a scaffold immune microenvironment did not correspond with

254 these clusters either (Fig. 5B) (12). It was determined that Ly6c2, Arg1, Mrc1 and

255 Nos2 were sufficient to distinguish the computationally determined AIM, PIM and

256 Mono subsets. We performed flow cytometry on cells isolated from the Aligned and

257 Ctrl samples using Cd68 (a monocyte-macrophage marker, also expressed by some

258 neutrophils and dendritic cells) and the proposed markers (Ly6c2, Arg1, Mrc1 and

259 Nos2). The $\mathrm{Cd} 68^{+}$cells were selected to create a t-distributed stochastic neighbor

260 embedding (tSNE) plot. We then identified $\operatorname{Arg} 1^{+}$macrophages expressing the surface

261 markers Mrc1 and Nos2 in the gated dataset to represent AIM and PIM, respectively.

262 The $\operatorname{Arg} 1^{+}$Ly $6 \mathrm{c} 2^{+}$monocytes were also identified (Mono). The three terminal clusters

263 (AIM, PIM, and Mono) could be separated, indicating that the subsets can be

264 identified experimentally using flow cytometry (Fig. 5C). Pseudo-temporal trajectory

265 (Monocle 2) of the four subsets revealed that monocytes developed into M-DCs and

266 polarized macrophages (AIM and PIM). Although AIM and PIM expressed distinct 
267 gene signatures, they were highly correlated (Fig. 5D-E). The Ctrl samples

268 contributed to a larger proportion of cells in all subsets (Fig. 5A). Therefore, the

269 scaffold might play a role in the reduction of macrophage infiltration at the

270 proliferative stage.

271 Sub-clustering of $\mathbf{T}$ cells revealed a novel $\mathbf{T}$ cell population and more effector $\mathbf{T}$

272 cells in the Aligned group

273 T cells were clustered into four subsets (Fig. 5F). T cells characterized by increased

$274 \mathrm{Cd} 7, \mathrm{Cd} 3 \mathrm{~g}$, and Areg were named as Early T cells (ET) (32). The subset adjacent to

275 ET expressed elevated Xc11 and Sult2b1 (associated with T cell activation), and was

276 named Activated T cell 1 (AT1) (33). AT1 also expressed increased Areg, Ctla2a, and

277 Ctla2b, genes related to immune homeostasis and immuno-suppression $(34,35)$.

278 Another activated T cell subset (AT2) expressed up-regulated genes associated with

279 cytotoxic T cells (Cd8b1), Th1 cells (Ifng, Ptpn18), and Th17 cells (Il17a, Il17f) (36,

280 37). Therefore, AT2 might include multiple effector $\mathrm{T}$ cells. A novel $\mathrm{T}$ cell subset

281 connecting AT2 and ET was featured by high expression of Birc5, Mki67 and Stmn1,

282 markers for cell proliferation, and was named Proliferating T cell (PT) (Fig. 5F-G). In

283 GO analysis, both ET and AT1 were enriched in T cell activation. AT1 was also found

284 elevation of $\mathrm{T}$ cell receptor signaling pathway, suggesting that these $\mathrm{T}$ cells played a

285 role in antigen recognition (Fig. 5G). We found three terminally differentiated clusters

286 stemming from two precursors (ET and AT1) in pseudo-time analysis. PT was

287 differentiated from AT1 and ET after the first branch point, and might be a transitional

288 status between early $\mathrm{T}$ cells and effector $\mathrm{T}$ cells. At the second branch, cells 
289

290

291

292

293

294

295

296

297

298

299

300

301

302

303

304

305

306

307

308

309

310

differentiated into two terminal clusters. One belonged to AT1 and ET, and another one was the AT2 population (Fig. 5H). Correlation analysis showed that ET and AT1 were highly correlated (Fig. 5I). In the ET and AT1 populations, more cells were from Ctrl samples, whereas a larger number of cells in AT2 and PT were from aligned samples.

\section{Receptor-ligand analysis reveals intricate interactions among immune cells,} keratinocytes and fibroblasts

To explore potential interactions among immune cells, keratinocytes and fibroblasts, we ran CellChat analysis on these datasets (38). For anti-inflammatory macrophages (AIM), broadly speaking, "pro-inflammatory” signals like Tnf, Visfatin, Rankl, EGFR and C3a signaling, and "anti-inflammatory" signals including Spp1, Lgals9, Sema3, Chemerin, Il2, Il13 and Il10 signaling were involved either in a paracrine or autocrine way. Signals like Mif, Ccl, Csf, Edn, and Cxcl12 signaling exerted protective or deleterious effects depending on their specific roles (Fig. 6E) $(25,39-50)$. Among T cell populations (Fig. 6F), ET communicated with other T cells mainly through Tgfb signaling, mediating ET cell tolerance and immune homeostasis (51). The novel PT population secreted Wnt11 signals that bound to Fzd5 receptor on ET. Inner bulge layer keratinocytes secreted Cxc116 that targeted Cxcr6 on ET, chemoattracting ET to the wound area (52). For fibroblast populations, HDF1 had the most frequent interaction with other cells (Fig. 6G). In addition to the mentioned signals, OSM signaling was also activated and induced the expression of the pro-inflammatory cytokine IL-6 (53). T cells and macrophages secreted Tgfb1 and Tgfb2 to target 
311 HDF1 and PF1, leading to proliferation and differentiation of fibroblasts into

312 myo-fibroblasts (54). Among keratinocytes, IFEB and IB1 had the most frequent

313 contact with other cells (Fig. 6H, Fig. S8I-M). IFEB received Tgfb1 and Tgfb2

314 (signals playing key roles in regulating epithelial-to-mesenchymal transition (EMT)

315 for keratinocytes) from $\mathrm{T}$ cells, macrophages and germinative layer (GL)

316 keratinocytes (55). Interactive plots for other types of cells were summarized in Fig.

317 S8. The communication between immune cells and cutaneous cells was complicated,

318 and wound healing was the comprehensive results of numerous influencing factors

319 working together.

320 Discussion

321 For this study, we fabricated electrospun membranes with three types of surface

322 topography (Random, Aligned, and Latticed). The aligned membranes led to faster

323 wound healing, reduced fibrotic response and enhanced regeneration of cutaneous

324 appendages compared to other scaffolds. Meanwhile, the aligned membranes

325 exhibited immunomodulatory properties (Fig. 6A). Based on that, we generated

326 single-cell transcriptomes from wounded mouse skin to investigate the

327 microenvironment around scaffolds. The skin wound repair process was classically

328 divided into four phases: hemostasis (hours), inflammation (days), proliferation (1-2

329 weeks), and remodeling (>2 weeks). 7 days post-wounding was a transitional time

330 point when the innate immune response subsided and activity of adaptive immune

331 cells increased (10). In the Ctrl samples, the infiltration of T cells achieved a similar

332 extent with macrophages (Fig. 6B Overall distribution). However, in Aligned group, 
333 infiltrated macrophages were much fewer than $\mathrm{T}$ cells, and more terminally

334 differentiated effector $\mathrm{T}$ cells were present (Fig. 6B). According to the timeframe of

335 innate and adaptive immune responses (10), the process of innate immunity seemed to

336 be alleviated earlier, and adaptive immune response was advanced in the presence of

337 aligned scaffolds (Fig. 6C). In the immune microenvironment around aligned

338 scaffolds (Fig. 6D), damage-associated molecular patterns (DAMPs),

339 pathogen-associated molecular patterns (PAMPs) and antigens from cell debris,

340 pathogens, and foreign agents (scaffold) triggered innate and adaptive immune

341 responses. Neutrophils from circulation $(\mathrm{PBN})$ quickly migrated to the wound area.

342 The infiltrated neutrophils (Neu1 and Neu2) phagocytosed dying cells and

343 microorganisms, and secreted chemo-attractants like Ccr1 and Ccl4 to recruit more

344 leukocytes and lymphoid cells (52). Circulating monocytes (Mono) were also

345 recruited to the wound area, and differentiated into pro-inflammatory macrophages

346 (PIM), clearing dying neutrophils and debris. Meanwhile, anti-inflammatory

347 macrophages (AIM) chemo-attracted more cells via chemokines like Ccl8 and Ccl6.

348 Part of monocytes differentiated into dendritic cells (M-DC). Tissue resident

349 Langerhans cells (LC), together with M-DC, functioned as antigen-presenting cells

350 (APCs). In the antigen specific signal, LC and DC processed antigens into peptides,

351 and presented them by MHC class II molecules (H2-Aa, H2-Eb1) on the cell surface.

352 T cells (ET and AT1) bound to the MHC molecules through surface receptors (TCR),

353 and differentiated into effector T cells ( $\mathrm{Cd} 4^{+} \mathrm{AT} 2$ cells). The co-stimulatory signal was

354 characterized by the engagement of $\mathrm{Cd} 28$ receptor on $\mathrm{T}$ cells (AT2, $\mathrm{Cd} 8 \mathrm{~b} 1^{+} \mathrm{Cd} 28^{\mathrm{hi}}$ ) 
355 with Cd86 ligands on $\mathrm{APCs}\left(\mathrm{Cd} 86^{+} \mathrm{DC}\right.$ and $\left.\mathrm{LC}\right)(10,56)$. With regard to the

356 influences that immune microenvironment had on tissue generation, immune cells

357 sent out a variety of signals (like Grn, Tgfb, Areg/Hbegf) that modulated behaviors of

358 keratinocytes and fibroblasts (Fig. 6). On the other hand, keratinocytes and fibroblasts

359 secreted chemotactic, pro- or anti-inflammatory signals that regulated immune cell

360 polarization and function. Immune cells themselves also interact with each other via

361 numerous signals. The communication network of immune cells and cutaneous cells

362 was complicated, and wound healing was the comprehensive results of these factors. 


\section{Acknowledgement}

365 This work was supported by Research and Develop Program, West China Hospital of

366 Stomatology Sichuan University (No.LCYJ2019-19); The Fundamental Research

367 Funds for The Central Universities (No. 2082604401239); National Key Research and

368 Development Program of China (No. 2016YFA0201703/2016YFA0201700)

369 Statement of conflict of interest

370 There are no conflicts of interest related to this manuscript. 


\section{Fig. 1}
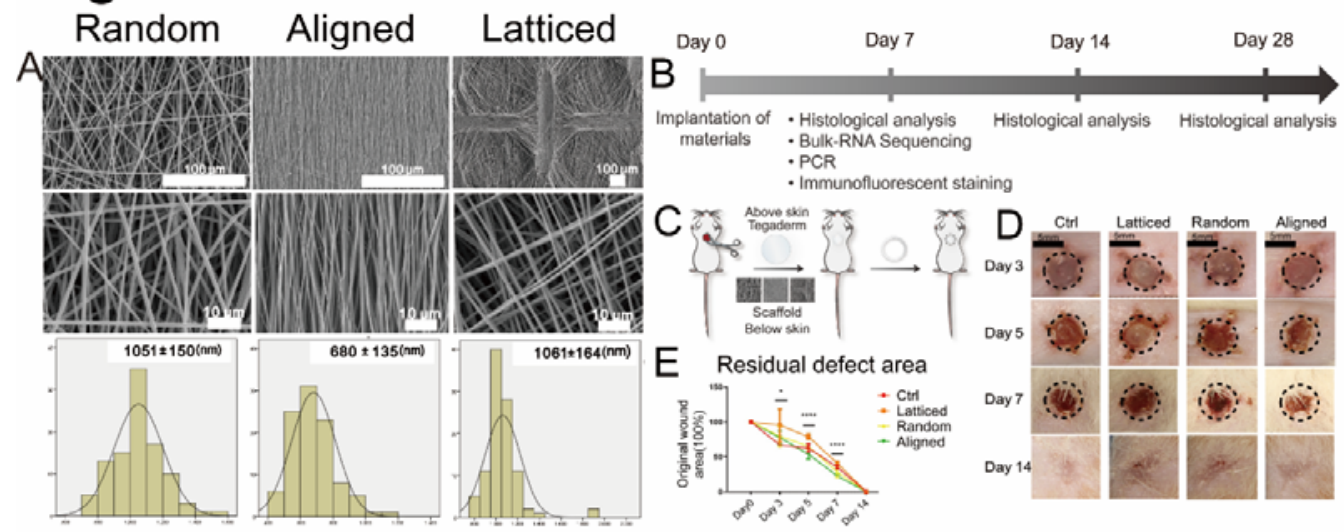

$\mathrm{F}$

$14 d$

$28 d$

G Gap width
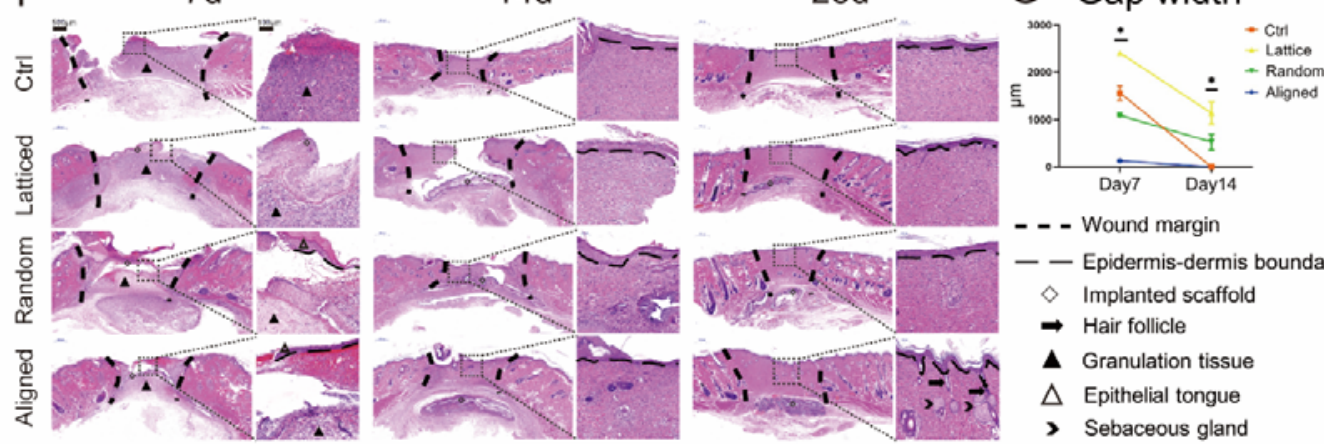

- - Wound margin

- - Epidermis-dermis boundary

$\diamond$ Implanted scaffold

$\rightarrow$ Hair follicle

A Granulation tissue

$\triangle$ Epithelial tongue

> Sebaceous gland

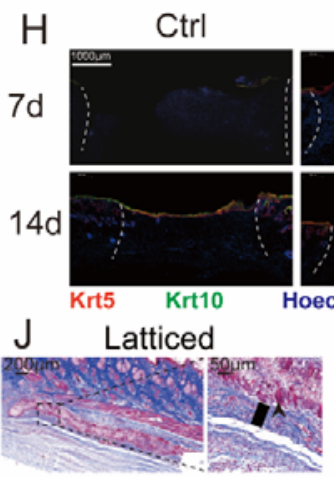

Latticed

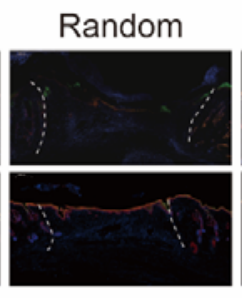

Aligned
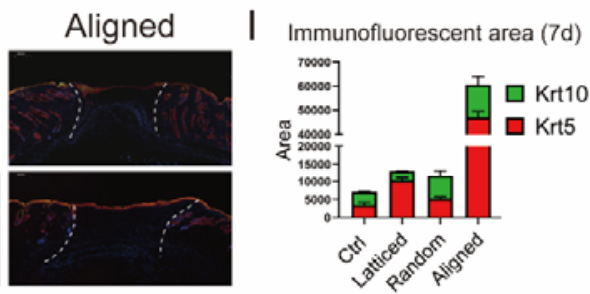

K Thickness of fibrotic capsule
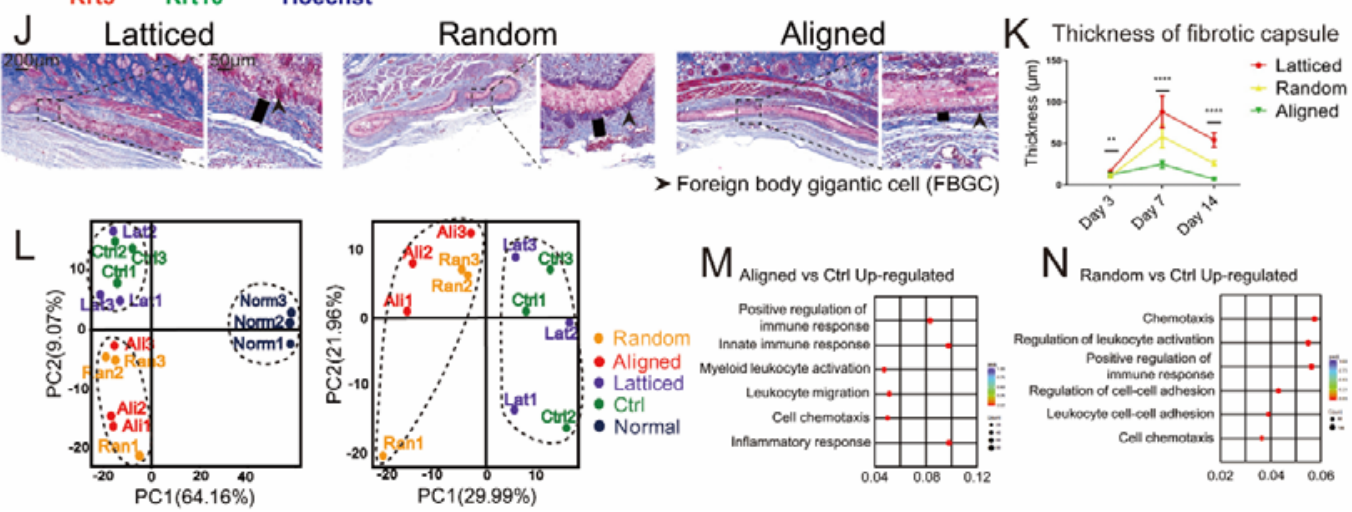

$\mathrm{N}$

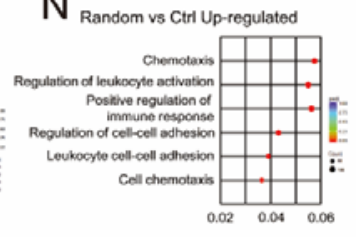

Fig. 1 Evaluation of rat skin wound healing implanted with three types of 
376 types of electrospun membranes. (B) Workflow for evaluating rat skin wound healing.

377 (C) Surgical processes for the rat skin excisional wound model. (D, E) Residual

378 wound area at each time point. (F) Histological view of Ctrl group and groups

379 implanted with three types of scaffolds. (G) Semi-quantitative evaluation of gap width.

$380 \quad(\mathrm{H})$ Immunofluorescent staining using Krt5 (red) and Krt10 (green). (I)

381 Semi-quantitative evaluation of the fluorescent area. (J) Evaluation of foreign body

382 reaction around biomaterials. FBGCs lined up on Random and Latticed membranes,

383 whereas fewer of them appeared on Aligned ones. (K) Thickness of fibrotic capsules.

384 (L-N) PCA and GO analysis revealed immunomodulatory effects for aligned and

385 random scaffolds. $* * * * \mathrm{P}<0.0001, * * \mathrm{P}<0.01$, and $* \mathrm{P}<0.05$ by ANOVA for data in

386 (E), (G) and (K).

387 
Fig. 2

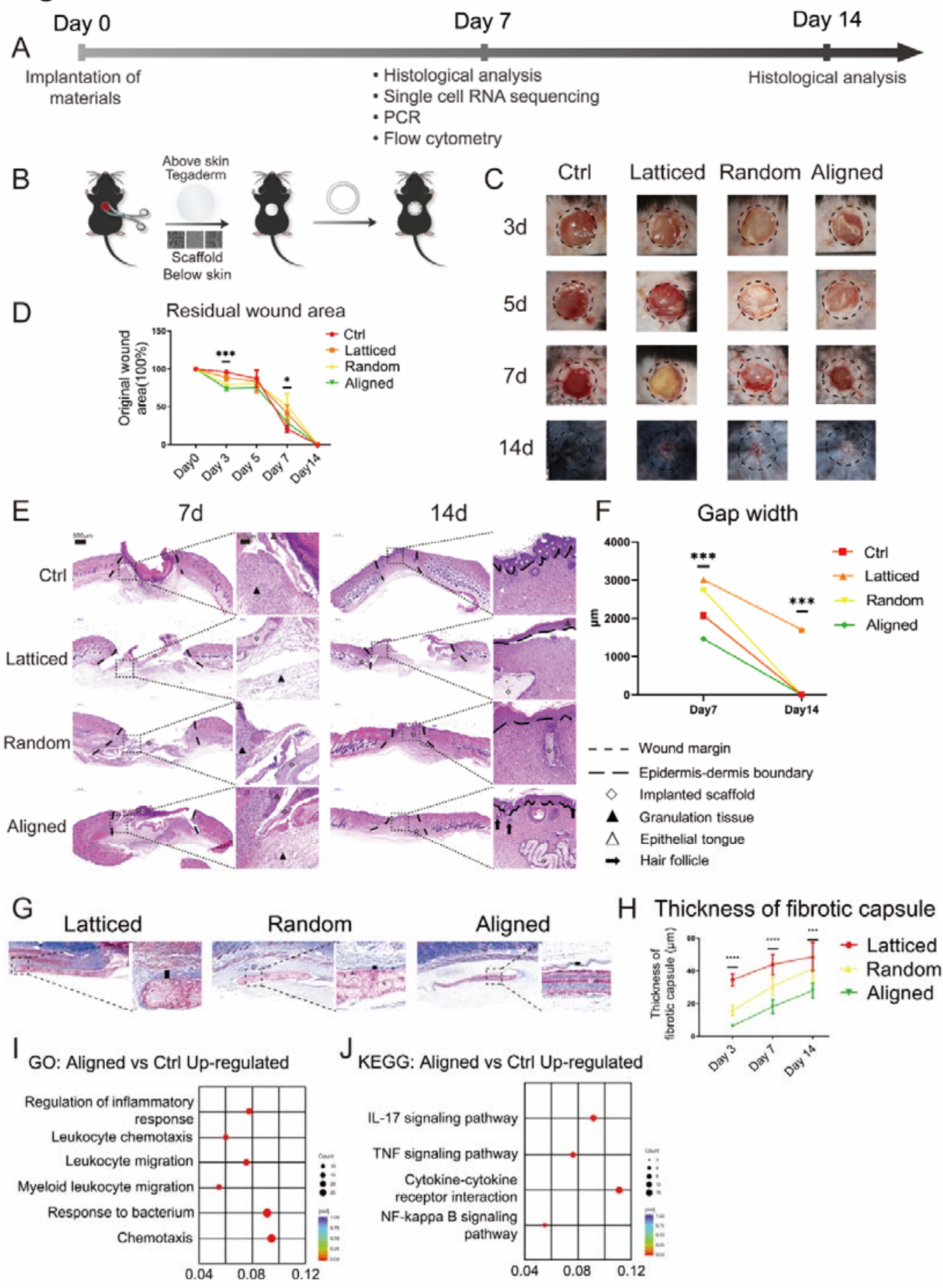

Fig. 2 Evaluation of mouse skin wound healing implanted with three types of

skin wound healing. (B) Surgical processes for mouse skin excisional wound model. 
392 (C, D) Residual wound area at each time point. (E) Histological analysis of Ctrl group

393 and groups implanted with three types of scaffolds. (F) Semi-quantitative evaluation

394 of gap width. (G) Evaluation of foreign body reaction around biomaterials. (H)

395 Thickness of fibrotic capsules. (I, J) GO and KEGG analysis revealed

396 immunomodulatory effects for aligned scaffolds. $* * * * \mathrm{P}<0.0001$, *** $\mathrm{P}<0.001$, and

$397 * \mathrm{P}<0.05$ by ANOVA for data in $(\mathrm{D}),(\mathrm{F})$ and $(\mathrm{H})$. 
Fig. 3

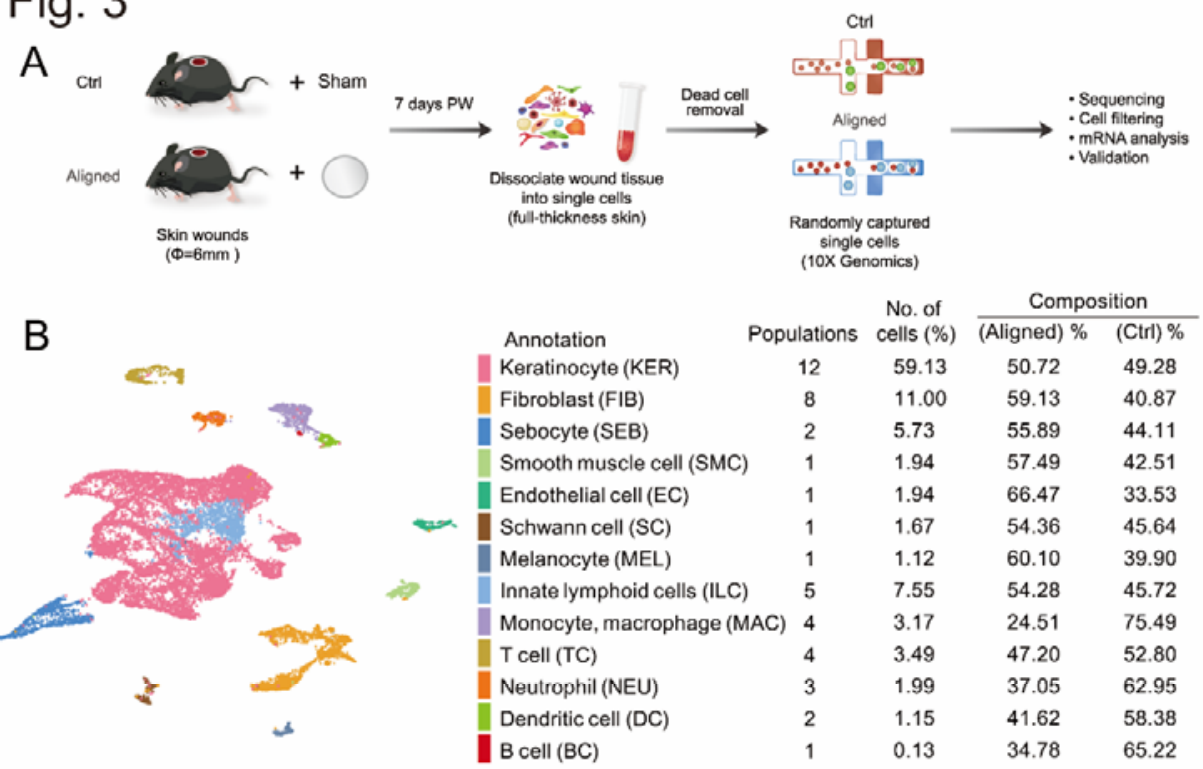

C
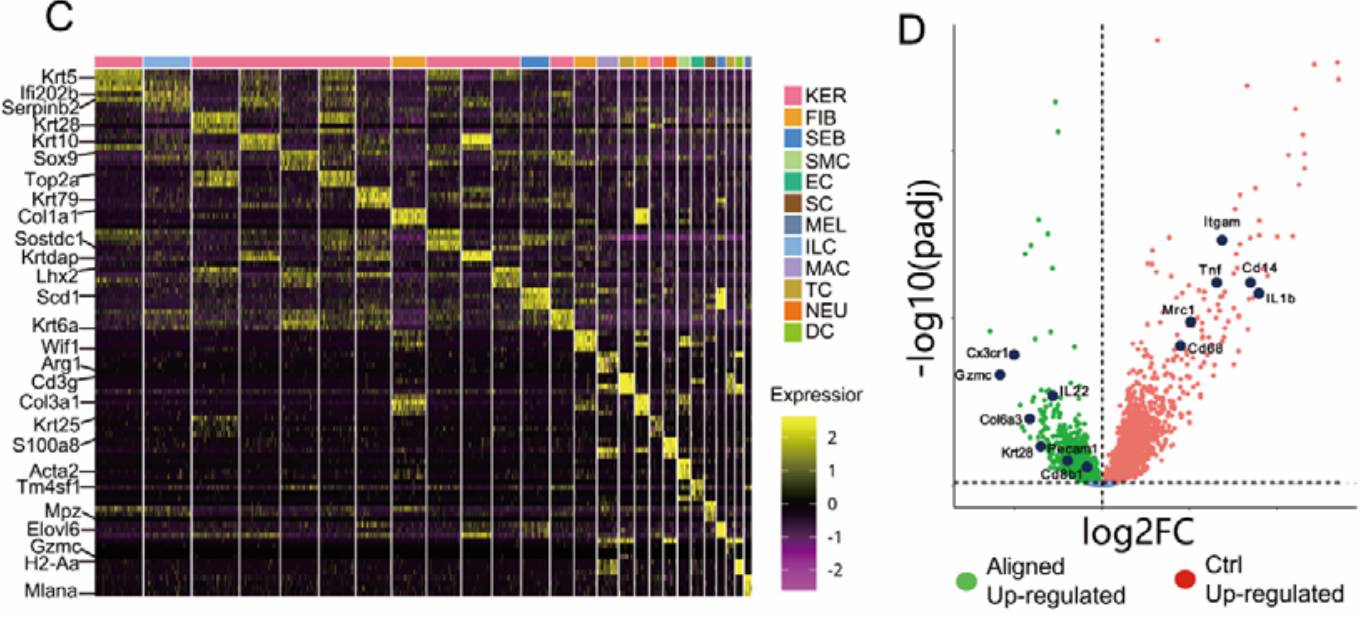

Fig. 3 Overview of the single-cell transcriptome analysis. (A) Workflow for

401 single-cell experiment. (B) Cells were categorized into thirteen main classes. Cell

402 populations in each class, number of cells (\%), and composition of aligned and Ctrl

403 cells were listed. (C) Marker genes for different cell classes. (D) Gene expression

404 differences between Ctrl and Aligned groups. 
Fig. 4

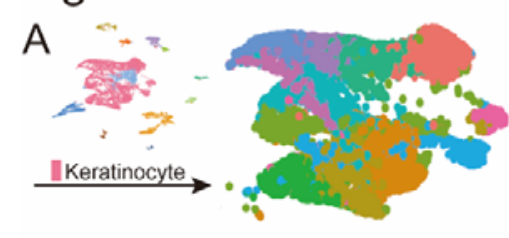

B
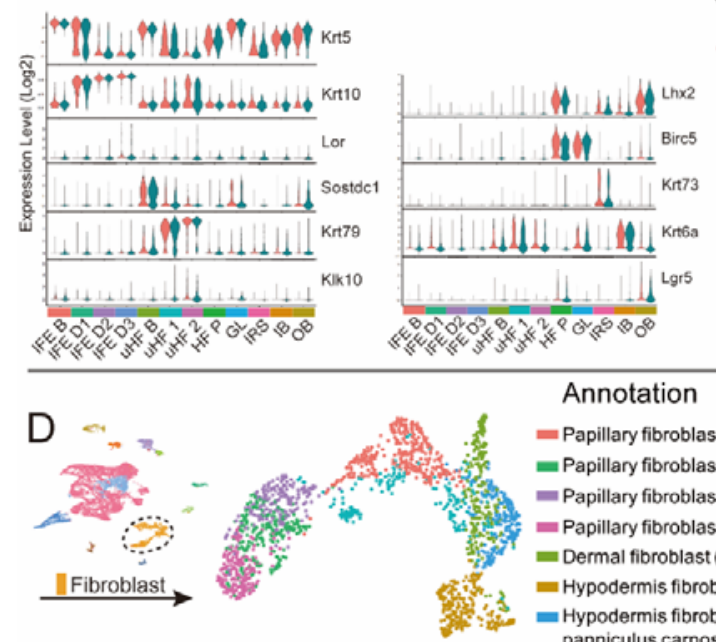

Annotation

$=$ Papillary fibroblast 1 (PF1)

$=$ Papillary fibroblast 2 (PF2)

$=$ Papillary fibroblast 3 (PF3)

- Papillary fibroblast 4 (PF4)

$=$ Dermal fibroblast (DF)

(Adventitia) (HDF1) Sfrp2, Plac8, Gpx3

- Hypodermis fibroblast 2 (Around the

panniculus carnosus muscle) (HDF2)

- Myofibroblast (MF)

\section{Telogen $\quad$ Anagen} Den, Celli, Collat Birc5, Tagln, Acta2

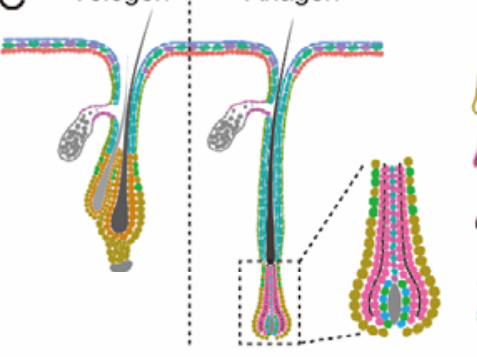

$\int$ Outer root sheath

$\bigwedge$ Inner root sheath

$\int$ Cortex/Cuticle

\Nedulla

A Germinative layer

\section{Markergenes Composition}

Htra1, Fmod, Crabp1 Col23a1, Wif1, Cald1 Prir, Hspb1, Col23a1 Vcan, Col23a1, Crabp1

Pcolce2, Gpx3, Den

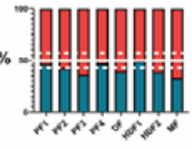

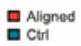

Marker genes Composition

$\begin{array}{llll}E & & \text { Annotation } & \text { Marker genes }\end{array}$

$F_{\text {Cst3r }}^{\text {PBN marker genes }} \quad$ Function (Top GO)

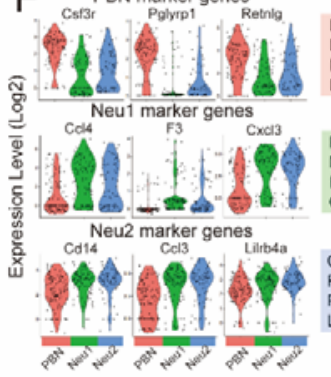

eukocyte chemotaxis Leukocyte migration
Neutrophil migration Defense response to other organism

Positive regulation of chemotaxis Myeloid leukocyte migration Regulation of chemotaxis Granulocyte chemotaxis

Cellular response to lipopolysaccharide Response to bacterium Response to molecule of bacterial origin Response to molecule of bacterial orign
LPS-mediated signaling pathway

G

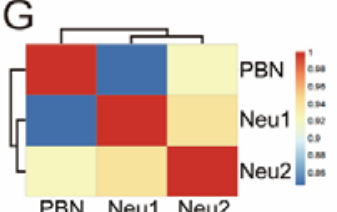

PBN Neu1 Neu2

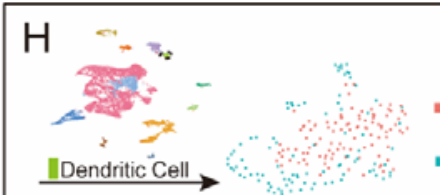

Annotation

- Monocyte-derived

dendritic cells (DC)

cells (LC)

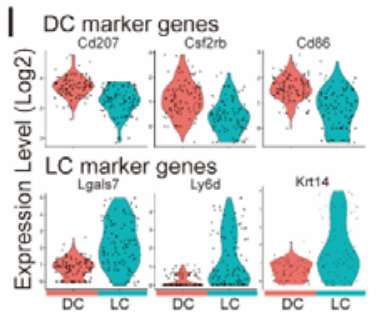

Marker genes

= Cd207, Csf2rb, Cd86, Rgs1 - Lgals7, Ly6d, Krt14, Krt5

Composition

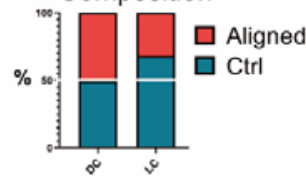

$\mathrm{J}$

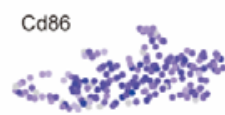

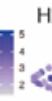

- Epidermal Langerhans

Fig. 4 Further analysis of keratinocytes, fibroblasts, neutrophils and dendritic

cells. (A) Sub-clustering of keratinocytes revealed twelve subsets. Marker genes and 
410 (C) Location of different keratinocyte subsets. Color of each cell corresponds with its

411 cell type in (A). (D) Sub-clustering of fibroblasts revealed eight subsets. The marker

412 genes and composition for each subset was listed. (E) Sub-clustering of neutrophils

413 revealed three subsets. (F) Marker genes for neutrophil subsets and their enriched

414 gene sets in GO analysis. (G) Correlation analysis of neutrophil subsets. (H)

415 Sub-clustering of dendritic cells revealed two subsets. (I) Marker genes for dendritic

416 cell subsets. (J) Expression of genes associated with antigen presenting in dendritic

417 cells. 
Fig. 5

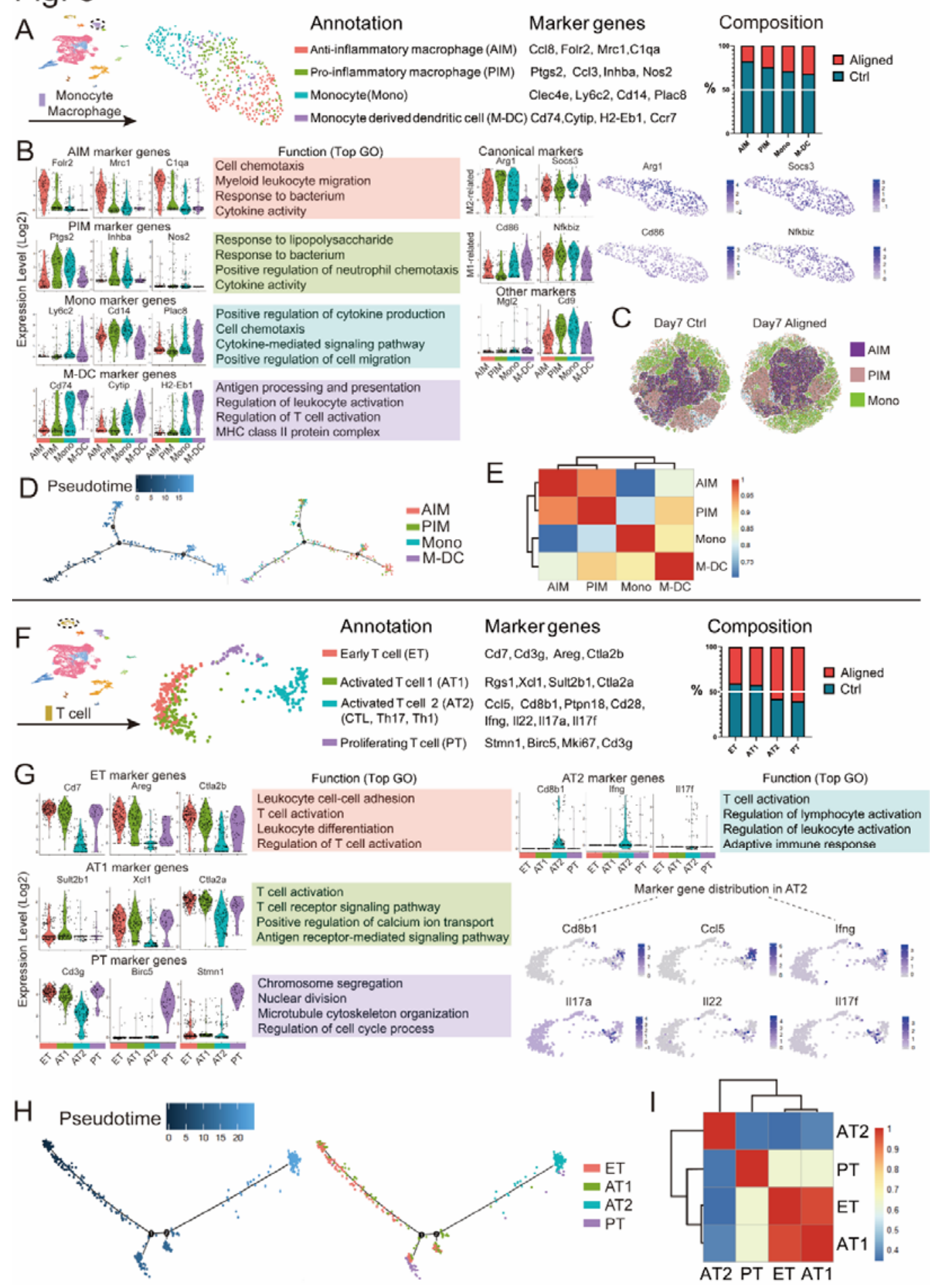

Fig. 5 Further analysis of macrophages and $\mathbf{T}$ cells. (A) Sub-clustering of

421 macrophages revealed four subsets. Marker genes and composition for each subset

422 was listed. (B) Marker genes for each macrophage/monocyte subset and their enriched 
423 gene sets in GO analysis. Expression of canonical M1 and M2 markers and other

424 proposed markers were shown. (C) In vivo flow cytometry strategy using Cd68 to

425 identify monocytes and macrophages. Arg1+ macrophages expressing the surface

426 markers Mrc1 and Nos2 in the gated dataset represented AIM and PIM, respectively.

427 The Arg1+Ly6c2+ monocytes were also identified (Mono). (D) Pseudo-temporal

428 ordering of macrophage/monocytes, and the distribution of four subsets along the

429 trajectory. (E) Correlation analysis of macrophage/monocyte subsets. (F)

430 Sub-clustering of $\mathrm{T}$ cells revealed four subsets. (G) Marker genes for $\mathrm{T}$ cell subsets

431 and their enriched gene sets in GO analysis. AT2 subset expressed gene signatures of

432 cytotoxic T cells, Th1 cells, and Th17 cells. (H) Pseudo-temporal ordering of T cells,

433 and the distribution of four subsets along the trajectory. (I) Correlation analysis of $\mathrm{T}$

434 cell subsets. 
Fig. 6

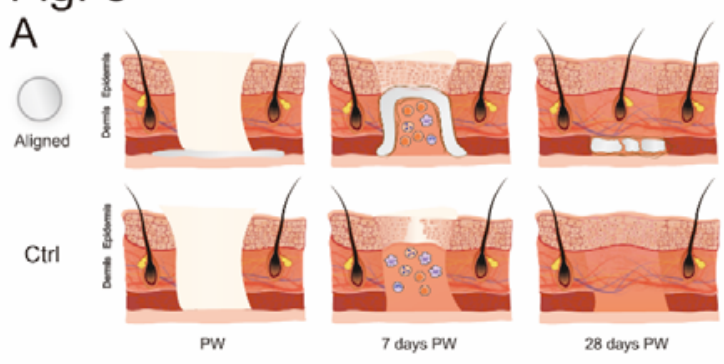

B Overall distribution

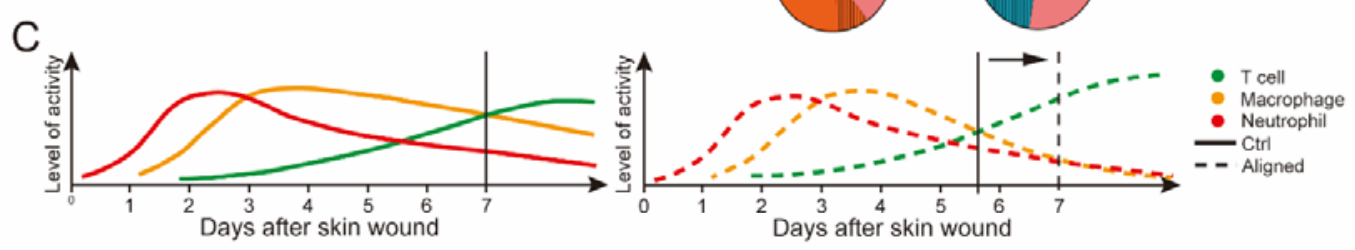

D
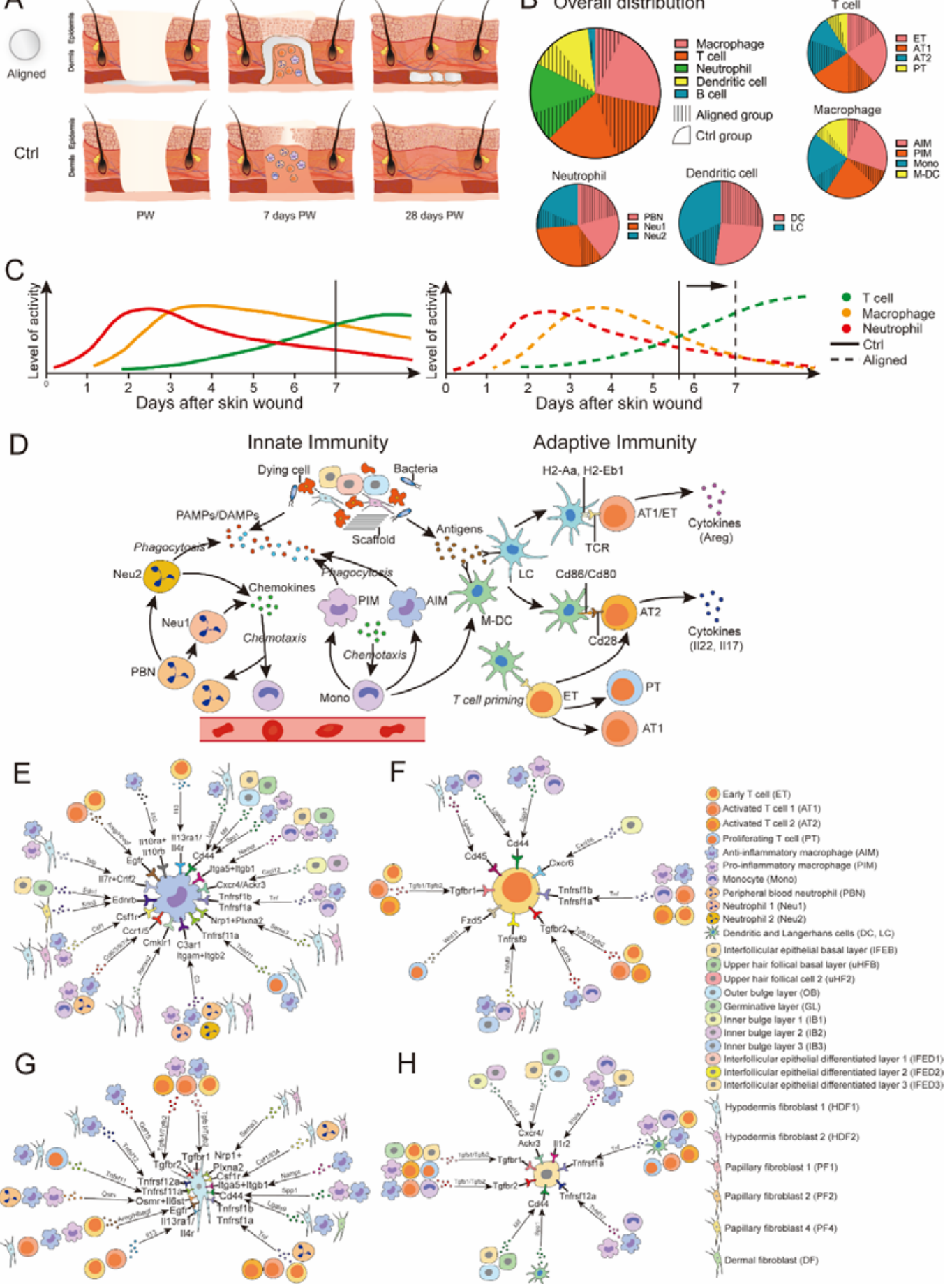

G

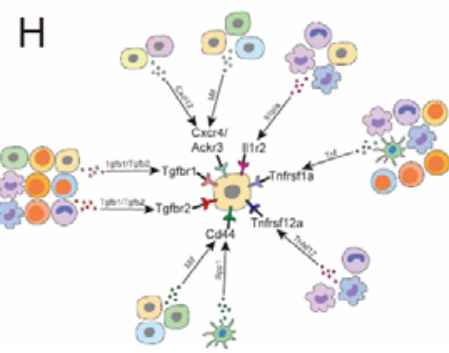

Fig. 6 Summary of the differences in wound healing and immune responses

between the Aligned group and Ctrl group. (A) The aligned membranes had 
440 response and enhanced regeneration of skin appendages. (B) Pie plot for overall

441 immune cells in two groups. For each cell type, differences in cell amount between

442 aligned and Ctrl groups. (C) Timeframe of innate and adaptive immune responses

443 occurred in Aligned and Ctrl groups. (D) The immune microenvironment around

444 aligned scaffolds. (E) Cell-cell interaction plots according to receptor-ligand analysis

445 via CellChat.

446 


\section{References}

448 1. X. Li, B. Cho, R. Martin, M. Seu, C. Zhang, Z. Zhou, J. S. Choi, X. Jiang, L.

449 Chen, G. Walia, J. Yan, M. Callanan, H. Liu, K. Colbert, J. 450 Morrissette-McAlmon, W. Grayson, S. Reddy, J. M. Sacks, H. Q. Mao, 451 Nanofiber-hydrogel composite-mediated angiogenesis for soft tissue 452 reconstruction. Sci Transl Med 11, eaau6210 (2019).

453 2. A. Mandal, A. V. Boopathy, L. K. W. Lam, K. D. Moynihan, M. E. Welch, N. 454 R. Bennett, M. E. Turvey, N. Thai, J. H. Van, J. C. Love, P. T. Hammond, D. J. 455 Irvine, Cell and fluid sampling microneedle patches for monitoring 456 skin-resident immunity. Sci Transl Med 10, eaar2227 (2018).

457 3. G. Huang, F. Li, X. Zhao, Y. Ma, Y. Li, M. Lin, G. Jin, T. J. Lu, G. M. Genin, F. $458 \mathrm{Xu}$, Functional and Biomimetic Materials for Engineering of the 459 Three-Dimensional Cell Microenvironment. Chem Rev 117, 12764-12850 (2017).

4. H. Li, Y. Xu, H. Xu, J. Chang, Electrospun membranes: control of the structure and structure related applications in tissue regeneration and drug delivery. J Mater Chem B 2, 5492-5510 (2014).

464 5. Y. Wang, H. Shi, J. Qiao, Y. Tian, M. Wu, W. Zhang, Y. Lin, Z. Niu, Y. Huang, 465 Electrospun tubular scaffold with circumferentially aligned nanofibers for 466 regulating smooth muscle cell growth. ACS Appl Mater Interfaces 6, 2958-2962 (2014). 
Neves, The influence of patterned nanofiber meshes on human mesenchymal stem cell osteogenesis. Macromol Biosci 11, 978-987 (2011).

4717. K. T. Kurpinski, J. T. Stephenson, R. R. Janairo, H. Lee, S. Li, The effect of fiber alignment and heparin coating on cell infiltration into nanofibrous PLLA scaffolds. Biomaterials 31, 3536-3542 (2010). C. Chu, L. Liu, S. Rung, Y. Wang, Y. Ma, C. Hu, X. Zhao, Y. Man, Y. Qu, Modulation of foreign body reaction and macrophage phenotypes concerning microenvironment. J Biomed Mater Res A 108, 127-135 (2020). Design, clinical translation and immunological response of biomaterials in regenerative medicine. Nat Rev Mater 1, 16040 (2016). strategies for local immunomodulation. Nat Rev Mater 4, 379-397 (2019). T. Wolf, A. J. Tam, I. Vanderzee, X. Wang, J. I. Andorko, H. Zhang, R. Narain, 12, eaax3799 (2020). 
macrophages drive IL-17-mediated fibrosis. Sci Immunol 4, eaax4783 (2019).

492

493

494

495

496

497

498

499

500

501

502

503

504

505

506

507

508

509

510

511

512

13. T. Stuart, A. Butler, P. Hoffman, C. Hafemeister, E. Papalexi, W. M. Mauck, 3rd, Y. Hao, M. Stoeckius, P. Smibert, R. Satija, Comprehensive Integration of Single-Cell Data. Cell 177, 1888-1902 e1821 (2019).

14. H. Wu, S. Han, B. Wu, X. Du, Z. Sheng, J. Lin, X. Chen, K. Zhao, V. Bunpetch, Y. Chen, M. Zeng, E. V. Alakpa, Y. Ma, X. Lei, J. Huang, X. Zou, H. Ouyang, Single-cell mass cytometry reveals in vivo immunological response to surgical biomaterials. Appl Mater Today 16, 169-178 (2019).

15. I. Pastar, O. Stojadinovic, N. C. Yin, H. Ramirez, A. G. Nusbaum, A. Sawaya, S. B. Patel, L. Khalid, R. R. Isseroff, M. Tomic-Canic, Epithelialization in Wound Healing: A Comprehensive Review. Adv Wound Care (New Rochelle) 3, 445-464 (2014).

16. R. Tokunaga, W. Zhang, M. Naseem, A. Puccini, M. D. Berger, S. Soni, M. McSkane, H. Baba, H. J. Lenz, CXCL9, CXCL10, CXCL11/CXCR3 axis for immune activation - A target for novel cancer therapy. Cancer Treat Rev 63, 40-47 (2018).

17. P. Myung, M. Ito, Dissecting the bulge in hair regeneration. J Clin Invest 122, 448-454 (2012).

18. S. Joost, K. Annusver, T. Jacob, X. Sun, T. Dalessandri, U. Sivan, I. Sequeira, R. Sandberg, M. Kasper, The Molecular Anatomy of Mouse Skin during Hair Growth and Rest. Cell Stem Cell 26, 441-457.e447 (2020).

19. C. Philippeos, S. B. Telerman, B. Oules, A. O. Pisco, T. J. Shaw, R. Elgueta, G. 

Dermatol 138, 802-810 (2018).

21. C. F. Guerrero-Juarez, P. H. Dedhia, S. Jin, R. Ruiz-Vega, D. Ma, Y. Liu, K. Yamaga, O. Shestova, D. L. Gay, Z. Yang, K. Kessenbrock, Q. Nie, W. S. Pear,

22. W. M. Nauseef, N. Borregaard, Neutrophils at work. Nat Immunol 15, 602-611 (2014).

24. Q. Su, B. Z. Igyarto, Keratinocytes Share Gene Expression Fingerprint with Epidermal Langerhans Cells via mRNA Transfer. J Invest Dermatol 139, 
polarization. Front Immunol 5, 402 (2014).

536 26. S. A. Islam, D. S. Chang, R. A. Colvin, M. H. Byrne, M. L. McCully, B.

537 Moser, S. A. Lira, I. F. Charo, A. D. Luster, Mouse CCL8, a CCR8 agonist, 538 promotes atopic dermatitis by recruiting IL-5+ T(H)2 cells. Nat Immunol 12, $539 \quad 167-177(2011)$.

540 27. M. Carrion, S. Perez-Garcia, C. Martinez, Y. Juarranz, L. Estrada-Capetillo, A. 541 Puig-Kroger, R. P. Gomariz, I. Gutierrez-Canas, VIP impairs acquisition of the 542 macrophage proinflammatory polarization profile. J Leukoc Biol 100, $543 \quad 1385-1393(2016)$.

544 28. J. E. Kanter, F. Kramer, S. Barnhart, M. M. Averill, A. Vivekanandan-Giri, T. 545 Vickery, L. O. Li, L. Becker, W. Yuan, A. Chait, K. R. Braun, S. Potter-Perigo, 546 S. Sanda, T. N. Wight, S. Pennathur, C. N. Serhan, J. W. Heinecke, R. A. 547 Coleman, K. E. Bornfeldt, Diabetes promotes an inflammatory macrophage 548 phenotype and atherosclerosis through acyl-CoA synthetase 1. Proc Natl Acad 549 Sci U S A 109, E715-724 (2012).

550 29. M. Clement, G. Basatemur, L. Masters, L. Baker, P. Bruneval, T. Iwawaki, M. 551 Kneilling, S. Yamasaki, J. Goodall, Z. Mallat, Necrotic Cell Sensor Clec4e 552 Promotes a Proatherogenic Macrophage Phenotype Through Activation of the 553 Unfolded Protein Response. Circulation 134, 1039-1051 (2016).

554 30. A. M. Soler-Rodriguez, H. Zhang, H. S. Lichenstein, N. Qureshi, D. W. Niesel, 555 S. E. Crowe, J. W. Peterson, G. R. Klimpel, Neutrophil activation by bacterial lipoprotein versus lipopolysaccharide: differential requirements for serum and 

Soulier, N. Boissel, T. Leblanc, A. Baruchel, J. Landman-Parker, P. H. Roméo, lymphoblastic leukemia discriminates functionally heterogeneous cell populations. Leukemia 25, 1249-1258 (2011). LXR signaling couples sterol metabolism to proliferation in the acquired immune response. Cell 134, 97-111 (2008). helper cells synthesizing steroids de novo to contribute to immune homeostasis. Cell Rep 7, 1130-1142 (2014). 
579 36. P. Castro-Sanchez, R. Ramirez-Munoz, P. Roda-Navarro, Gene Expression Profiles of Human Phosphotyrosine Phosphatases Consequent to Th1 Polarisation and Effector Function. J Immunol Res 2017, 8701042 (2017).

37. I. C. Boothby, J. N. Cohen, M. D. Rosenblum, Regulatory T cells in skin injury: At the crossroads of tolerance and tissue repair. Sci Immunol 5, eaaz9631 (2020).

38. S. Jin, C. F. Guerrero-Juarez, L. Zhang, I. Chang, P. Myung, M. V. Plikus, Q. Nie, Inference and analysis of cell-cell communication using CellChat. bioRxiv, (2020).

39. M. Croft, The role of TNF superfamily members in T-cell function and diseases. Nat Rev Immunol 9, 271-285 (2009). 
601

602

603

604

605

606

607

608

609

610

611

612

613

614

615

616

617

618

619

620

621

622
I. D. Mascanfroni, Z. Al Adham, S. Lavoie, M. Ibourk, D. D. Nguyen, J. N.

Samsom, J. C. Escher, R. Somech, B. Weiss, R. Beier, L. S. Conklin, C. L.

Ebens, F. G. Santos, A. R. Ferreira, M. Sherlock, A. K. Bhan, W. Muller, J. R.

Mora, F. J. Quintana, C. Klein, A. M. Muise, B. H. Horwitz, S. B. Snapper,

Interleukin-10 receptor signaling in innate immune cells regulates mucosal

immune tolerance and anti-inflammatory macrophage function. Immunity $\mathbf{4 0 ,}$ 706-719 (2014).

44. S. S. Jankauskas, D. W. L. Wong, R. Bucala, S. Djudjaj, P. Boor, Evolving complexity of MIF signaling. Cell Signal 57, 76-88 (2019).

45. W. Lin, D. Xu, C. D. Austin, P. Caplazi, K. Senger, Y. Sun, S. Jeet, J. Young, D. Delarosa, E. Suto, Z. Huang, J. Zhang, D. Yan, C. Corzo, K. Barck, S. Rajan, C. Looney, V. Gandham, J. Lesch, W. C. Liang, E. Mai, H. Ngu, N. Ratti, Y. Chen, D. Misner, T. Lin, D. Danilenko, P. Katavolos, E. Doudemont, H. Uppal, J. Eastham, J. Mak, P. E. de Almeida, K. Bao, A. Hadadianpour, M. Keir, R. A. D. Carano, L. Diehl, M. Xu, Y. Wu, R. M. Weimer, J. DeVoss, W. P. Lee, M. Balazs, K. Walsh, K. R. Alatsis, F. Martin, A. A. Zarrin, Function of CSF1 and IL34 in Macrophage Homeostasis, Inflammation, and Cancer. Front Immunol 10, 2019 (2019).

46. S. F. Nie, L. F. Zha, Q. Fan, Y. H. Liao, H. S. Zhang, Q. W. Chen, F. Wang, T. T. Tang, N. Xia, C. Q. Xu, J. Y. Zhang, Y. Z. Lu, Z. P. Zeng, J. Jiao, Y. Y. Li, T. Xie, W. J. Zhang, D. Wang, C. C. Wang, J. J. Fa, H. B. Xiong, J. Ye, Q. Yang, P. Y. Wang, S. H. Tian, Q. L. Lv, Q. X. Li, J. Qian, B. Li, G. Wu, Y. X. Wu, Y. 
Yang, X. P. Yang, Y. Hu, Q. K. Wang, X. Cheng, X. Tu, Genetic Regulation of the Thymic Stromal Lymphopoietin (TSLP)/TSLP Receptor (TSLPR) Gene Expression and Influence of Epistatic Interactions Between IL-33 and the TSLP/TSLPR Axis on Risk of Coronary Artery Disease. Front Immunol 9, 1775 (2018).

47. S. Avniel, Z. Arik, A. Maly, A. Sagie, H. B. Basst, M. D. Yahana, I. D. Weiss, B. Pal, O. Wald, D. Ad-El, N. Fujii, F. Arenzana-Seisdedos, S. Jung, E. Galun, E. Gur, A. Peled, Involvement of the CXCL12/CXCR4 pathway in the recovery of skin following burns. J Invest Dermatol 126, $468-476$ (2006).

48. A. Stofkova, Resistin and visfatin: regulators of insulin sensitivity, inflammation and immunity. Endocr Regul 44, 25-36 (2010).

49. D. M. Hardbower, K. Singh, M. Asim, T. G. Verriere, D. Olivares-Villagomez, D. P. Barry, M. M. Allaman, M. K. Washington, R. M. Peek, Jr., M. B. Piazuelo, K. T. Wilson, EGFR regulates macrophage activation and function in bacterial infection. J Clin Invest 126, 3296-3312 (2016).

50. M. C. Walsh, Y. Choi, Biology of the RANKL-RANK-OPG System in Immunity, Bone, and Beyond. Front Immunol 5, 511 (2014).

51. J. C. Marie, D. Liggitt, A. Y. Rudensky, Cellular mechanisms of fatal early-onset autoimmunity in mice with the $\mathrm{T}$ cell-specific targeting of transforming growth factor-beta receptor. Immunity 25, 441-454 (2006).

52. S. K. Bromley, T. R. Mempel, A. D. Luster, Orchestrating the orchestrators: chemokines in control of T cell traffic. Nat Immunol 9, 970-980 (2008). 
645 53. B. Le Goff, S. Singbrant, B. A. Tonkin, T. J. Martin, E. Romas, N. A. Sims, N.

646 C. Walsh, Oncostatin M acting via OSMR, augments the actions of IL-1 and TNF in synovial fibroblasts. Cytokine 68, 101-109 (2014).

648 54. V. Miguel, O. Busnadiego, M. Fierro-Fernandez, S. Lamas, Protective role for miR-9-5p in the fibrogenic transformation of human dermal fibroblasts. Fibrogenesis Tissue Repair 9, 7 (2016).

55. S. Liarte, A. Bernabe-Garcia, F. J. Nicolas, Human Skin Keratinocytes on Sustained TGF-beta Stimulation Reveal Partial EMT Features and Weaken Growth Arrest Responses. Cells 9, 255 (2020).

56. M. L. Kapsenberg, Dendritic-cell control of pathogen-driven T-cell polarization. Nat Rev Immunol 3, 984-993 (2003).

Materials and methods

\section{Electrospinning of polymer scaffolds}

We used poly(lactic-co-glycolic acid) (PLGA) (LA/GA = 75:25, Mw = $105 \mathrm{kDa}$,

663 (Aladdin Co., Ltd. (Shanghai, China)) were loaded into a plastic syringe fitted with a 664 flat-tipped $21 \mathrm{G}$ needle (inner diameter $=0.5 \mathrm{~mm}$ ). A high voltage of $7 \mathrm{kV}$ and a distance 665 of $16 \mathrm{~cm}$ were applied between the needle and the collector. For randomly oriented 
667 grounded flat collector in the high electric field, forming a membrane deposited on the 668 aluminium foil. For the Latticed group, an electroconductive chess-like wire net was 669 used as the collector. For the Aligned group, the rotational speed of the collecting 670 drum is set at $2800 \mathrm{rpm}$. Finalized scaffolds were approximately $30-90 \mu \mathrm{m}$ in 671 thickness. To crosslink FC, the membranes were immersed in $50 \mathrm{mM}$ of EDS/NHS 672 and $10 \mathrm{mM}$ of MES ethanol solution for $24 \mathrm{~h}$ at $4{ }^{\circ} \mathrm{C}$. Then membranes were washed 673 three times with $75 \%$ ethanol and dried in vacuum oven for $24 \mathrm{~h}$. Subsequently, the 674 prepared membranes were sterilized by $\gamma$-irradiation for in vitro and in vivo 675 experiments.

\section{Characterization of scaffolds}

677 Scanning electron microscopy (SEM; JEOL, JSM-6510LV, Japan) was employed to 678 observe the surface morphology of the electrospun membranes. Image-Pro Plus was 679 applied to quantitatively measure the fiber diameter and distribution from the SEM 680 images obtained. The surface wetting behavior of the membranes were characterized 681 by measuring the water contact angles (Chengde Dingsheng, JY-82B, China). Five 682 samples were tested for each type of membrane to obtain an average value. The 683 tensile properties of the membranes were tested under a constant upper clamp at speed 684 of $15 \mathrm{~mm} / \mathrm{min}$. All tensile tests follow the criteria of "Plastics-Determination of tensile properies of films" (GB/T 1040.3-2006, corresponding with ISO 1184-1983).

\section{Cell culture and cell viability test}

687 L929 mouse fibroblast cells and Human oral keratinocytes (HOK) were used for 
689

690

691

692

693

694

695

696

697

698

699

700

701

702

703

704

705

706

707

708

709

710

(HyClone) supplemented with $10 \%$ fetal bovine serum (Gibco), and were kept at $37 \square$ in humidified 5\% CO2/95\% air. The cell viability was determined by Cell Counting Kit-8 (CCK-8, Dojindo Laboratories, Kumamoto, Japan). Electrospun membranes were cut into squares (edge length $=5 \mathrm{~mm}$ ) and placed in the bottom of 96 -well plates ( $n=3$ for each group). L929 cells and HOK cells were seeded onto membranes at $4 * 10^{4}$ cells $/ \mathrm{ml}$. Cells were co-cultured with membranes for 1,3 , and 5 days. Blank wells seeded with equal amount of cells were used as Ctrl. $10 \mu \mathrm{lCCK}-8$ solution was added to each well, and the plates were incubated at $37^{\circ} \mathrm{C}$ for $1 \mathrm{~h}$. After incubation, the Abs at 450nm was measured to determine the cell viability using a micro-plate reader (Multiskan, Thermo, USA).

\section{Experimental model}

\section{Excisional wound model}

The protocol of the present experiment was approved by Institution Review Board of West China Hospital of Stomatology (No.WCHSIRB-D-2017-033-R1) Animals included Sprague Dawley male rat at ages from 7 to 8 weeks and C57BL/6 male mice at ages from 7 to 9 weeks (Chengdu Dossy Experimental Animals Co., LTD.). Hair at the surgical area was removed. Full-thickness circular excisional wound (diameter $=6 \mathrm{~mm}$ ) was created at the dorsal skin of rats/mice. Random, aligned and latticed electrospun scaffolds were trimmed into circular shape (diameter $=8 \mathrm{~mm})$, and placed below the wound. The Control group did not receive any implants. A sterile Tegaderm film (3M) was placed above the wound to protect the wound area. Then annular silicone splints (inner diameter $=8 \mathrm{~mm}$, outer diameter $=12 \mathrm{~mm}$ ) were sutured 
711 with the Tegaderm film and underlying skin in order to minimize the contraction of

712 the dorsal muscle. After healing for 1,2 and 4 weeks, animals were euthanized for

713 sample harvest. Using the residual wound as center, a round skin sample (diameter

$714=10 \mathrm{~mm}$ ) containing all the layers of skin was harvested.

715 Model for subcutaneous implant placement

716 The surgical area on dorsal skin was shaved and aseptically prepared. Three

717 horizontal incisions of approximately $10 \mathrm{~mm}$ were made and subcutaneous pockets

718 were created for membrane implantation. Then random, align and lattice scaffolds

719 were placed into the pockets. After implantation, the incisions were sutured with

720 interrupted sutures. After recovering for 3, 7, and 14 days, samples including scaffolds

721 and the whole layer of skin at surgical sites were together harvested.

722 Specimen harvest for scRNA-seq

723 We obtained skin samples by cutting off skin at the wound area (circular,

724 diameter $=10 \mathrm{~mm})$. Subcutaneous tissues were removed. A total of four samples were

725 harvested in each group. The tissues were washed in a $100 \mathrm{~mm}$ petri dish containing

$72620 \mathrm{ml}$ of phosphate-buffered saline (PBS). Then they were transferred to a $50 \mathrm{~mm}$

727 petri dish containing $100 \mu \mathrm{L}$ of Enzyme G (Epidermis Dissociation Kit mouse,

728 Miltenyi) and $3.9 \mathrm{ml}$ of PBS buffer with the dermal side facing downwards. Tissues

729 were digested for 16 hours at $4^{\circ} \mathrm{C}$. Then they were transferred into a $50 \mathrm{~mm}$ petri dish

730 containing $4 \mathrm{~mL}$ of $1 \times$ Buffer $\mathrm{S}$ (Miltenyi). Epidermis was peeled off from the skin

731 using tweezers, and was cut into pieces. Enzyme mix containing $3.9 \mathrm{ml}$ of $1 \times$ Buffer S,

$732100 \mu \mathrm{l}$ of Enzyme P, and $20 \mu \mathrm{l}$ of Enzyme A (Miltenyi) stored in a gentleMACS ${ }^{\mathrm{TM}} \mathrm{C}$ 
733 Tube was used to digest the epidermis pieces for 20 minutes at $37 \square$. Then $4 \mathrm{ml}$ of

734 PBS that contained $0.5 \%$ bovine serum albumin (BSA) was added. A gentleMACS

735 Dissociator (Miltenyi) was applied to automatically dissociate the epidermis (Program

736 B). The sample was passed through a $70 \mu \mathrm{m}$ cell strainer (Corning), centrifuged at

$737300 \times \mathrm{g}$ for 10 minutes at room temperature, and resuspended with PBS that contained

$7380.5 \%$ BSA. Cells were gently washed twice and stored in an ice box. For the dermis

739 part, they were first cut into pieces (diameter $<1 \mathrm{~mm}$ ). The tissue was mixed with

$74010 \mathrm{ml}$ enzyme mix containing Type I Collagenase $(3125 \mathrm{u} / \mathrm{mL})($ Gibco) and $2.5 \mathrm{ml}$

741 trypsin (Gibco), and poured into a gentleMACS ${ }^{\mathrm{TM}} \mathrm{C}$ Tube. After dissociating the

742 tissue on gentleMACS Dissociator for 37s (Skin mode), another 10ml enzyme mix

743 was added. The sample was digested for 2.5 hours at $37 \square$ in a rotary machine

744 (Peqlab). Then the dermis sample was passed through a $70 \mu \mathrm{m}$ cell strainer (Corning),

745 centrifuged at $300 \times \mathrm{g}$ for 5 minutes at room temperature, and resuspended with $3 \mathrm{ml}$

746 red blood cell lysis buffer (Solarbio). After 3 minutes, the cell suspension was

747 centrifuged and gently resuspended with RPMI 1640 medium (Hyclone). Cells were

748 gently washed twice with PBS containing 0.5\% BSA and stored in an ice box. The

749 epidermis and dermis cell solutions were mixed together as a whole. The sample was

750 centrifuged, and resuspended with $100 \mu \mathrm{l}$ Dead Cell Removal MicroBeads (Miltenyi).

751 After incubation for $15 \mathrm{~min}$ at room temperature, the cell suspension was diluted in

$7523 \mathrm{ml} 1 \times$ Binding buffer (Miltenyi). LS columns (Miltenyi) and a magnetic stand

753 (Miltenyi) were used for removal of dead cells and debris. The negatively selected

754 live cells passed through the column, and were resuspended with PBS containing $0.05 \%$ 
755 BSA. Finally, we proceeded with the 10x Genomics ${ }^{\circledR}$ Single Cell Protocol.

756 Single-cell encapsulation and library generation

757 Single cells were encapsulated in water-in-oil emulsion along with gel beads coated

758 with unique molecular barcodes using the 10x Genomics Chromium Single-Cell

759 Platform. For single-cell RNA library generation, the manufacturers' protocol was

760 performed. (10×Single Cell 3' v3) Sequencing was performed using Illumina 1.9

761 mode with 94574 reads per cell.

762 Quality control

763 We use fastp to analyze basic statistics on the quality of the raw reads.

764 If clean reads is indispensable, then raw read sequences produced by the Illumina

765 pipeline in FASTQ format were pre-processed through Trimmomatic software which

766 can be summarized as below:

767 (1)Remove low-quality reads: scan the reads with a 4-base wide sliding window, cut

768 when the average quality per base drops below 10 (SLIDINGWINDOW: 4:10)

769 (2)Remove trailing low quality or $\mathrm{N}$ bases (below quality 3) (TRAILING: 3)

770 (3)Remove adapters: there are two modes to remove the adapter sequence: a.

771 alignment with the adapter sequence, the number of matching bases was greater than

7727 and mismatch=2; b. when read1 and read2 overlapping base scoring was greater

773 than 30, we removed non-overlapping portions (ILLUMINACLIP:adapter.fa:2:30:7)

774 (4)Drop reads below the 26 bases long

775 (5)Discard those reads that did not form pairs.

776 The remaining reads that passed all the filtering steps was counted as clean reads and 
777 all subsequent analyses were based on this.

778 Generation and Analysis of Single-Cell Transcriptomes

779 Raw reads were demultiplexed and mapped to the reference genome by $10 \mathrm{X}$

780 Genomics Cell Ranger pipeline

781 (https://support.10xgenomics.com/single-cell-gene-expression/software/pipelines/late

782 st/what-is-cell-ranger) using default parameters. All downstream single-cell analyses

783 were performed using Seurat. In brief, for each gene and each cell barcode (filtered by

784 CellRanger3.0), unique molecule identifiers were counted to construct digital

785 expression matrices. Secondary filtration by Seurat: A gene with expression in more

786 than 3 cells was considered as expressed, and each cell was required to have at least

787200 expressed genes. We also filtered out some of the foreign cells.

788 The Seurat package was used in data normalization, dimension reduction, clustering,

789 and differential expression. We used the Seurat alignment method canonical

790 correlation analysis (CCA) [Nat. Biotechnol. 36, 411-420 (2018).] for integrated

791 analysis of datasets. For clustering, highly variable genes were selected and the

792 principal components based on those genes used to build a graph, which was

793 segmented with a resolution of 0.6.

\section{$794 \quad$ RNA-seq analysis}

795 The bulk tissue RNA-seq analysis was carried out by Novogene Corporation (Beijing,

796 China). RNA was extracted from tissues or cells using standard methods to make sure

797 samples were strictly controlled for quality. In terms of library construction and

798 quality control, mRNA can be obtained in two main ways: firstly, most eukaryotes' 
799

800

801

802

803

804

805

806

807

808

809

810

811

812

813

814

815

816

817

818

819

820

mRNA has poly A-tailed structural, and poly A-tailed mRNA can be enriched by Oligo (dT) magnetic beads. The other is the removal of ribosomal RNA from the total RNA to obtain mRNA. Subsequently, the obtained mRNA was randomly interrupted by divalent cations in NEB Fragmentation Buffer, and the database was constructed according to the NEB general database construction method or chain specific database construction method. Upon completion of library construction, a Qubit2.0 Fluorometer was used for initial quantification, and the library was diluted to $1.5 \mathrm{ng} / \mathrm{ul}$. Then the insert size of the library was detected using Agilent 2100 bioanalyzer. After the insert size met the expectation, the effective concentration of the library was accurately quantified by qRT-PCR (the effective concentration of the library higher than $2 \mathrm{nM}$ ) to ensure library quality. Finally, the libraries were qualified for sequencing, and Illumina sequencing was performed after pooling the different libraries according to the requirements of effective concentration and target data volume, of which the basic principle is Sequencing by Synthesis. Through z-transformation of Fragments Per Kilobase of transcript per Million mapped reads (fpkm) of the selected gene, gene expression was analyzed. Sample size for conventional, bulk RNA-Seq libraries was fixed at 3 biological replicates.

\section{Quantitative Real-Time Polymerase Chain Reaction (qPCR).}

The harvested samples were cut into pieces, and homogenated in TRIzol ${ }^{\mathrm{TM}}$ Reagent (Cat. \#15596026, Invitrogen, Thermo Scientific). Concentration and ratio of total RNA were detected by NanoPhotometer NP80 (Implen, Westlake Village, CA) at wavelength of $260 \mathrm{~nm}$ and $280 \mathrm{~nm}$. The cDNAs were synthesized using 
821 PrimeScript ${ }^{\mathrm{TM}}$ RT reagent Kit with gDNA Eraser (Perfect Real Time) (Cat. 822 \#RR047A), then amplified by qPCR with the specific primers (Table 1). PCR was 823 performed on QuantStudio 3 Real-Time PCR Systems (ThermoFisher Scientific, 824 Waltham, MA). Each $20 \mu \mathrm{L}$ of PCR mixture contained $10 \mu$ l of TB Green Premix Ex 825 Taq (Tli RNaseH Plus, Takara) (2X), $0.4 \mu \mathrm{L}$ of PCR Forward Primer $(10 \mu \mathrm{M}), 0.4 \mu \mathrm{l}$ of 826 PCR Reverse Primer $(10 \mu \mathrm{M}), 0.4 \mu \mathrm{l}$ of ROX Reference Dye (50X), $2 \mu \mathrm{l}$ Template and $8276.8 \mu \mathrm{l}$ of Sterile purified water. Samples were incubated at 1 cycle of $95 \square$ for $30 \mathrm{~s}$ 828 followed by 40 cycles of $95 \square$ for $5 \mathrm{~s}$ and $60 \square$ for $34 \mathrm{~s}$, and ended up with a cycle 829 composing of $95 \square$ for $15 \mathrm{~s}, 60 \square$ for $1 \mathrm{~min}$ and $95 \square$ for $15 \mathrm{~s}$. Results were analyzed 830 using the comparative $\mathrm{CT}(\Delta \Delta \mathrm{CT})$ method to calculate gene expression fold changes 831 normalized to the levels of Gapdh gene transcripts. The experiments were repeated for 832 three times independently $(n=3)$.

\begin{tabular}{|l|l|l|l|}
\hline Primer & Forward sequence & Primer & Reverse sequence \\
\hline $\begin{array}{l}\text { Gapdh } \\
\text { forward }\end{array}$ & AGTGCCAGCCTCGTCTCATA & $\begin{array}{l}\text { Gapdh } \\
\text { reverse }\end{array}$ & GATGGTGATGGGTTTCCCGT \\
\hline $\begin{array}{l}\text { Cd68 } \\
\text { forward }\end{array}$ & TGGGGCCTCTCTGTATTG & $\begin{array}{l}\text { Cd68 } \\
\text { reverse }\end{array}$ & TCTGATGTCGGTCCTGTTT \\
\hline $\begin{array}{l}\text { Tgm2 } \\
\text { forward }\end{array}$ & CGGAAGGACACGGTTAGG & $\begin{array}{l}\text { Tgm2 } \\
\text { reverse }\end{array}$ & GTATGGGACCAGGAAACCC \\
\hline $\begin{array}{l}\text { Cd4 } \\
\text { forward }\end{array}$ & AACCAAAAGGTGTCTGTGC & $\begin{array}{l}\text { Cd4 } \\
\text { reverse }\end{array}$ & ACCAGCAAACTGAAGGGA \\
\hline $\begin{array}{l}\text { Cd3e } \\
\text { forward }\end{array}$ & TCTCCATCTCAGGAACCAG & $\begin{array}{l}\text { Cd3e } \\
\text { reverse }\end{array}$ & CAGGCAGGACTTTGTCATT \\
\hline
\end{tabular}

Table1 TB Green rat mRNA primers

834 Fluorescence activated Cell Sorting (FACS) analysis

835 The surface markers of macrophages and their phenotypes were examined by flow

836 cytometry to evaluate proportion and polarization of macrophages.

837 The in vivo specimens were first cut into pieces (diameter $<1 \mathrm{~mm}$ ). The tissue was 
838

839

840

841

842

843

844

845

846

847

848

849

850

851

852

853

mixed with $10 \mathrm{ml}$ enzyme mix containing Type I Collagenase (3125u/mL) (Gibco) and $2.5 \mathrm{ml}$ trypsin (Gibco), and poured into a gentleMACS ${ }^{\mathrm{TM}} \mathrm{C}$ Tube. After dissociating the tissue on gentleMACS Dissociator for 37s (Skin mode), another 10ml enzyme mix was added. The sample was digested for $2.5-3$ hours at $37 \square$ in a rotary machine (Peqlab). Then sample was passed through a 70 $\mu \mathrm{m}$ cell strainer (Corning), centrifuged at $300 \times \mathrm{g}$ for 5 minutes at room temperature. Cells were gently washed twice with PBS containing $0.05 \%$ BSA and stored in an ice box. Then the cell solutions were co-incubated with antibodies against iNOS (PE, NBP2-22119PE, Novus), CD68 (FITC, ab134351, Abcam), Arg1 (PE/Cyanine7, Cat\# 369707, Biolegend), Ly6c (Alexa Fluor 700, Cat\# 128023, Biolegend) and Mrc1 (Alexa Fluor 647, ab195192, Abcam) at 1:400 dilution in the dark for $1 \mathrm{~h}$ at $4^{\circ} \mathrm{C}(100 \mu \mathrm{l}$ per antibody for each sample). All samples were centrifuged at $450 \mathrm{RCF}$ for $5 \mathrm{~min}$ at $4^{\circ} \mathrm{C}$. Supernatants were removed by aspiration, $1 \mathrm{ml} 1 \mathrm{XPBS}$ solution containing $0.05 \%$ BSA was used to wash the cells twice. FACS analysis was performed on NovoCyte Flow Cytometers (ACEA Biosciences ${ }^{\circledR}$, San Diego, California) and FlowJo10.5.0. The experiments were repeated for three times independently $(n=3)$.

\section{Histological and immunofluorescent staining}

The sections were pretreated with $1 \%$ BSA in PBS containing $0.1 \%$ Triton X 100 for $1 \mathrm{~h}$, incubated in $1 \%$ Tween 20 for 20 min and washed again in PBS. The sections were subsequently analyzed for Krt10 and Krt5, according to the manufacturers' instructions. Briefly, sections were incubated for $30 \mathrm{~min}$ in dark. The excessive dye was rinsed off with PBS. Sections were incubated with antibody isotype to exclude 
860 false positive staining. Double immunofluorescence staining with primary antibodies

861 against cytokeratin 10 (ab76318, Abcam, 1:150), cytokeratin 5 (ab52635, Abcam,

862 1:200) and secondary antibodies (GB25303, GB21303, Servicebio, 1:400) was

863 performed. The immunostained specimens were further subjected to Hoechst 33258

864 staining (G1011, Servicebio). At least three parallel sections were observed with

865 fluorescence microscope (ZEISS SteREO Discovery.V20, Olympus). The

866 fluorescence area measurement was conducted on five random sights of regenerated

867 epithelium with CaseViewer 2.1 and Image Pro Plus $7.0(\mathrm{n}=5)$.

\section{Statistical Analysis}

869 Statistical significance for in vivo and in vitro data of histological analysis, qPCR and

870 FACS were analyzed by analysis of variance (ANOVA) at the 95\% confidence level,

871 which were performed in GraphPad Prism 8.0 (GraphPad Software, San Diego, CA,

872 USA) and $\mathrm{P}<0.05$ was considered statistically significant, while $\mathrm{P}>0.05$ was

873 considered having no statistical differences, which was marked with NS. 